\title{
Accelerating the knowledge of Peruvian
}

\section{Chalcididae (Insecta, Hymenoptera, Chalcidoidea) with integrative taxonomy}

\author{
Bruno Cancian de Araujo ${ }^{\ddagger}$, , Marcelo Teixeira Tavares $§$, Thales Renan Aguiar Brotto§, Juliana \\ Martins Silva-Freitas§, Max Estefani Vagner Santos ${ }^{\S}$, Pâmella Machado Saguiah§, Stefan Schmidt ${ }^{\ddagger}$ \\ ‡ SNSB-Zoologische Staatssammlung München, Munich, Germany \\ $\S$ Laboratório de Biodiversidade de Insetos, Universidade Federal do Espírito Santo, Vitória, Brazil
}

Corresponding author: Bruno Cancian de Araujo (chalcididae@gmail.com)

Academic editor: Matthew Yoder

Received: 03 May 2019 | Accepted: 02 Oct 2019 | Published: 04 Dec 2019

Citation: Cancian de Araujo B, Tavares MT, Brotto TRA, Silva-Freitas JM, Santos MEV, Saguiah PM, Schmidt S

(2019) Accelerating the knowledge of Peruvian Chalcididae (Insecta, Hymenoptera, Chalcidoidea) with integrative taxonomy. Biodiversity Data Journal 7: e35907. https://doi.org/10.3897/BDJ.7.e35907

ZooBank: urn:Isid:zoobank.org:pub:33893A19-DB0C-402F-866C-BE3C8C3BBED5

\section{Abstract}

We present the first regional inventory of the fauna of Chalcididae in the Peruvian Amazon, with a nearly 6 -fold increase in the number of species recorded for the country. A total of 418 specimens of Chalcididae were collected between 2000 and 2017 at the Panguana Reserve, Peruvian Amazon, 400 of which were obtained using Malaise traps and the remaining 18 specimens by canopy fogging. The morphological analyses indicated that these specimens represent 183 species of Chalcididae in 10 different genera, with 173 new to Peru and 134 potentially new species. We submitted 268 specimens, representing 167 species, to DNA barcoding. Of these, 141 specimens yielded sequences, 136 of them with a minimum of $300 \mathrm{bp}$. Sixty specimens were assigned a BIN by the Barcode of Life Database System (BOLD), resulting in 50 BINs. A cluster analysis of 138 individuals that yielded DNA sequences longer than $100 \mathrm{bp}$ revealed 118 MOTUs (molecular operative taxonomic units), all of them highly congruent with the morphological data. Prior to the present study, 37 species in 9 genera of Chalcididae were known from Peru. With our results, this number was increased to 210 species in 13 genera. The 
present study is the result of a joint effort between the SNSB - Zoologische Staatssammlung München, Germany (ZSM) and the Insect Biodiversity Laboratory of the Universidade Federal do Espírito Santo, Vitória, Brazil (LaBI-UFES), intending to apply an accelerated taxonomic treatment of the Chalcididae of the Panguana reserve using traditional morphological approaches in combination with DNA barcoding. The complete molecular dataset and associated voucher information is publicly available through BOLD. The new species that were discovered as part of the study are being formally described elsewhere as part of taxonomic treatments of Neotropical and world generic revisions at LaBI-UFES.

\section{Keywords}

Biodiversity, parasitoids, DNA barcoding, Peru, survey, species discovery

\section{Introduction}

Chalcididae is a medium-sized family with about 1,500 documented species in 87 genera and five subfamilies (Noyes 2018). Most individuals are between 1.5 and $24 \mathrm{~mm}$ in size (Tavares and Aquino 2014) and are recognised, amongst other characters, by the parallel inner margins of the compound eyes, rounded tegula, the lateral margin of the mesoscutum $\cap$-shaped and by their usually robust body with enlarged hind femora that are equipped with one or more teeth (or a comb of denticle) on the ventral margin.

Most of Chalcididae species are primary parasitoids of Lepidoptera and Diptera and a few species attack Coleoptera, Hymenoptera, Neuroptera or Strepsiptera (Tavares and Aquino 2014). Some species are secondary parasitoids (obligatory or facultative) through Hymenoptera (Dryinidae or Ichneumonoidea) and Diptera (Sarcophagidae or Tachinidae). The secondary hosts are Lepidoptera (many families), Coleoptera (Coccinellidae), Hemiptera (Auchenorrhyncha), Orthoptera (Acrididae) and Araneae (Theridiidae) (Tavares and Aquino 2014, Noyes 2018, Tavares et al. 2019). Although it is a family of parasitoid wasps that are moderately species-rich, the diversity of attacked hosts and of others biological aspects make the family contribute in a very diverse way to the structure and function of ecosystems. In addition, many species have shown effective or potential importance as natural enemies of pests (Delvare 2006).

Peru is one of the 17 megadiverse countries of the world (Mittermeier et al. 1999). However, only 38 species of Chalcididae have been recorded from the country so far (Noyes 2018), reflecting the lack of dedicated studies of chalcidoid fauna in that country (Table 1). 


\section{Table 1.}

Checklist of formally described species of Chalcididae known to occur in Peru. Taxa marked with $\left({ }^{*}\right)$ are new records for the country. A question mark (?) indicates that no region or place in the country was recorded.

\begin{tabular}{|c|c|c|c|c|}
\hline Subfamily & Tribe & scientificName & Location & references \\
\hline Chalcidinae & - & - & - & - \\
\hline - & Brachymeriini & - & - & - \\
\hline - & - & Brachymeria caudigera Bouček, 1992* & $\begin{array}{l}\text { Peruvian } \\
\text { Amazon }\end{array}$ & present study \\
\hline- & - & B. costalimai Delvare, 2017 & $\begin{array}{l}\text { Peruvian } \\
\text { Amazon }\end{array}$ & $\begin{array}{l}\text { Delvare et al. 2017, pg. } \\
36\end{array}$ \\
\hline - & - & B. flavipes (Fabricius, 1793) & ? & $\begin{array}{l}\text { Arias and Delvare 2003, } \\
\text { pg. } 129\end{array}$ \\
\hline - & - & B. mnestor (Walker, 1841)* & $\begin{array}{l}\text { Peruvian } \\
\text { Amazon }\end{array}$ & present study \\
\hline - & - & B. mochica Delvare, 2017 & Pacific Coast & $\begin{array}{l}\text { Delvare and Huchet } \\
2017 \text {, pg. } 46\end{array}$ \\
\hline - & - & B. pandora (Crawford, 1914)* & $\begin{array}{l}\text { Peruvian } \\
\text { Amazon }\end{array}$ & present study \\
\hline - & - & B. podagrica (Fabricius, 1787) & $\begin{array}{l}\text { Peruvian } \\
\text { Amazon }\end{array}$ & $\begin{array}{l}\text { Delvare and Huchet } \\
2017 \text {, pg. } 52\end{array}$ \\
\hline - & - & $\begin{array}{l}\text { Ceyxia acutigaster Andrade and Tavares, } \\
2009\end{array}$ & $\begin{array}{l}\text { Peruvian } \\
\text { Amazon }\end{array}$ & $\begin{array}{l}\text { Andrade and Tavares } \\
\text { 2009, pg. } 515\end{array}$ \\
\hline- & - & $\begin{array}{l}\text { Ce. amazonica Andrade and Tavares, } \\
2009^{*}\end{array}$ & $\begin{array}{l}\text { Peruvian } \\
\text { Amazon }\end{array}$ & present study \\
\hline- & - & $\begin{array}{l}\text { Ce. atuberculata Andrade and Tavares, } \\
2009\end{array}$ & $\begin{array}{l}\text { Peruvian } \\
\text { Amazon }\end{array}$ & $\begin{array}{l}\text { Andrade and Tavares } \\
\text { 2009, pg. } 518\end{array}$ \\
\hline- & - & Ce. bellissima Andrade and Tavares, $2009^{*}$ & $\begin{array}{l}\text { Peruvian } \\
\text { Amazon }\end{array}$ & present study \\
\hline- & - & Ce. concitator (Walker, 1862) & $\begin{array}{l}\text { Peruvian } \\
\text { Amazon }\end{array}$ & $\begin{array}{l}\text { Andrade and Tavares } \\
\text { 2009, pg. } 522\end{array}$ \\
\hline- & - & Ce. decreta (Walker, 1862) & $\begin{array}{l}\text { Peruvian } \\
\text { Amazon }\end{array}$ & $\begin{array}{l}\text { Andrade and Tavares } \\
2009 \text {, pg. } 523\end{array}$ \\
\hline- & - & Ce. flaviscapus Girault, 1911 & $\begin{array}{l}\text { Peruvian } \\
\text { Amazon }\end{array}$ & $\begin{array}{l}\text { Andrade and Tavares } \\
\text { 2009, pg. } 528\end{array}$ \\
\hline
\end{tabular}




\begin{tabular}{|c|c|c|c|c|}
\hline Subfamily & Tribe & scientificName & Location & references \\
\hline- & - & Ce. villosa (Olivier, 1791) & $\begin{array}{l}\text { Peruvian } \\
\text { Amazon }\end{array}$ & De Santis 1979, pg. 59 \\
\hline- & Chalcidini & - & - & - \\
\hline- & - & Conura abdominalis (Walker, 1862) & $?$ & $\begin{array}{l}\text { Arias and Delvare 2003, } \\
\text { pg. } 133\end{array}$ \\
\hline- & - & Co. acuta (Fabricius, 1804) & $?$ & Bouček 1992, pg. 13 \\
\hline- & - & Co. adela (Burks, 1939)* & $\begin{array}{l}\text { Peruvian } \\
\text { Amazon }\end{array}$ & present study \\
\hline- & - & Co. amoena (Say, 1836) & $\begin{array}{l}\text { Peruvian } \\
\text { Amazon* }\end{array}$ & $\begin{array}{l}\text { Arias and Delvare 2003, } \\
\text { pg. } 133\end{array}$ \\
\hline- & - & Co. attacta (Walker, 1864) & $\begin{array}{l}\text { Peruvian } \\
\text { Amazon* }\end{array}$ & Delvare 1992, pg. 133 \\
\hline- & - & Co. bidentata (Ashmead, 1904) ${ }^{*}$ & $\begin{array}{l}\text { Peruvian } \\
\text { Amazon }\end{array}$ & present study \\
\hline- & - & Co. camescens Delvare, $1992^{*}$ & $\begin{array}{l}\text { Peruvian } \\
\text { Amazon }\end{array}$ & present study \\
\hline- & - & Co. chapadae (Ashmead, 1904)* & $\begin{array}{l}\text { Peruvian } \\
\text { Amazon }\end{array}$ & present study \\
\hline- & - & Co. dares (Walker, 1842)* & $\begin{array}{l}\text { Peruvian } \\
\text { Amazon }\end{array}$ & present study \\
\hline- & - & Co. debilis (Say, 1836)* & $\begin{array}{l}\text { Peruvian } \\
\text { Amazon }\end{array}$ & present study \\
\hline- & - & Co. decisa (Walker, 1861) & $\begin{array}{l}\text { Peruvian } \\
\text { Amazon* }\end{array}$ & $\begin{array}{l}\text { Arias and Delvare 2003, } \\
\text { pg. } 134\end{array}$ \\
\hline- & - & Co. destinata (Walker, 1864) & $\begin{array}{l}\text { Peruvian } \\
\text { Amazon* }\end{array}$ & $\begin{array}{l}\text { Arias and Delvare 2003, } \\
\text { pg. } 134\end{array}$ \\
\hline- & - & Co. dorsimaculata (Cameron, 1884) & $\begin{array}{l}\text { Peruvian } \\
\text { Amazon }\end{array}$ & present study \\
\hline- & - & Co. eubule (Cresson, 1865) & $?$ & Delvare 1992, pg. 279 \\
\hline- & - & Co. emarginata (Fabricius, 1804) & $?$ & $\begin{array}{l}\text { Arias and Delvare 2003, } \\
\text { pg. } 134\end{array}$ \\
\hline- & - & Co. expleta (Walker, 1864) & $?$ & $\begin{array}{l}\text { Arias and Delvare } 2003 \text {, } \\
\text { pg. } 134\end{array}$ \\
\hline- & - & Co. femorata (Fabricius, 1775) & $\begin{array}{l}\text { Peruvian } \\
\text { Amazon* }\end{array}$ & $\begin{array}{l}\text { Arias and Delvare 2003, } \\
\text { pg. } 134\end{array}$ \\
\hline
\end{tabular}




\begin{tabular}{|c|c|c|c|c|}
\hline Subfamily & Tribe & scientificName & Location & references \\
\hline- & - & Co. ferruginea (Fabricius, 1804) & $\begin{array}{l}\text { Peruvian } \\
\text { Amazon* }\end{array}$ & $\begin{array}{l}\text { Arias and Delvare 2003, } \\
\text { pg. } 134\end{array}$ \\
\hline- & - & Co. flava (Fabricius, 1804) & $?$ & $\begin{array}{l}\text { Arias and Delvare 2003, } \\
\text { pg. } 134\end{array}$ \\
\hline- & - & Co. hirtifemora (Ashmead, 1885) & $?$ & $\begin{array}{l}\text { Arias and Delvare 2003, } \\
\text { pg. } 131\end{array}$ \\
\hline- & - & Co. immaculata (Cresson, 1865) & $\begin{array}{l}\text { Peruvian } \\
\text { Amazon* }\end{array}$ & Delvare 1997, pg. 131 \\
\hline- & - & Co. initia Delvare, 1997 & $\begin{array}{l}\text { Peruvian } \\
\text { Amazon }\end{array}$ & Delvare 1997, pg. 502 \\
\hline- & - & Co. juxta (Cresson, 1872) & $?$ & Delvare 1997, pg. 135 \\
\hline- & - & Co. laddi (Girault, 1913) & $?$ & $\begin{array}{l}\text { Arias and Delvare 2003, } \\
\text { pg. } 135\end{array}$ \\
\hline- & - & Co. maculipennis (Cameron, 1884) & $?$ & De Santis 1979, pg. 56 \\
\hline- & - & Co. masus (Walker, 1841) & $?$ & Delvare 1992, pg. 284 \\
\hline- & - & Co. miniata (Cameron, 1884)* & $\begin{array}{l}\text { Peruvian } \\
\text { Amazon }\end{array}$ & present study \\
\hline- & - & Co. mourei (De Santis, 1980)* & $\begin{array}{l}\text { Peruvian } \\
\text { Amazon }\end{array}$ & present study \\
\hline- & - & Co. nigrifrons (Cameron, 1884)* & $\begin{array}{l}\text { Peruvian } \\
\text { Amazon }\end{array}$ & present study \\
\hline- & - & Co. pygmaea (Fabricius, 1804)* & $\begin{array}{l}\text { Peruvian } \\
\text { Amazon }\end{array}$ & present study \\
\hline- & - & Co. rasplusi Delvare, $1992^{*}$ & $\begin{array}{l}\text { Peruvian } \\
\text { Amazon }\end{array}$ & present study \\
\hline- & - & Co. santaremensis (Ashmead, 1904)* & $\begin{array}{l}\text { Peruvian } \\
\text { Amazon }\end{array}$ & present study \\
\hline- & - & Co. tricolorata (Cameron, 1913) & $?$ & Delvare 1992, pg. 318 \\
\hline- & - & Co. vau (Ashmead, 1904)* & $\begin{array}{l}\text { Peruvian } \\
\text { Amazon }\end{array}$ & present study \\
\hline- & - & Co. vesicula Delvare, $1992^{*}$ & $\begin{array}{l}\text { Peruvian } \\
\text { Amazon }\end{array}$ & present study \\
\hline- & - & $\begin{array}{l}\text { Melanosmicra carenata Navarro-Tavares } \\
\text { and Tavares, } 2008^{*}\end{array}$ & $\begin{array}{l}\text { Peruvian } \\
\text { Amazon }\end{array}$ & present study \\
\hline
\end{tabular}




\begin{tabular}{|c|c|c|c|c|}
\hline Subfamily & Tribe & scientificName & Location & references \\
\hline- & - & Me. flavicollis (Cameron, 1904)* & $\begin{array}{l}\text { Peruvian } \\
\text { Amazon }\end{array}$ & present study \\
\hline- & - & Me. immaculata Ashmead, 1904* & $\begin{array}{l}\text { Peruvian } \\
\text { Amazon }\end{array}$ & present study \\
\hline- & - & $\begin{array}{l}\text { Me. nigra Navarro-Tavares and Tavares, } \\
2008^{*}\end{array}$ & $\begin{array}{l}\text { Peruvian } \\
\text { Amazon }\end{array}$ & present study \\
\hline- & - & $\begin{array}{l}\text { Me. tricolor Navarro-Tavares and Tavares, } \\
2008^{*}\end{array}$ & $\begin{array}{l}\text { Peruvian } \\
\text { Amazon }\end{array}$ & present study \\
\hline- & - & Stenosmicra sp. & $?$ & Bouček 1992, pg. 98 \\
\hline- & Phasgonophorini & - & - & - \\
\hline- & - & Stypiura batesii (Kirby, 1883)* & $\begin{array}{l}\text { Peruvian } \\
\text { Amazon }\end{array}$ & present study \\
\hline- & - & S. dentipes (Fabricius, 1804) & $?$ & $\begin{array}{l}\text { Arias and Delvare 2003, } \\
\text { pg. } 137\end{array}$ \\
\hline- & - & S. serripes (Fabricius, 1804) & $?$ & $\begin{array}{l}\text { Arias and Delvare 2003, } \\
\text { pg. } 138\end{array}$ \\
\hline Dirhininae & - & - & - & - \\
\hline- & Dirhinini & - & - & - \\
\hline- & - & Dirhinus buscki (Crawford, 1913)* & $\begin{array}{l}\text { Peruvian } \\
\text { Amazon }\end{array}$ & present study \\
\hline- & - & D. cameroni (Ashmead, 1904)* & $\begin{array}{l}\text { Peruvian } \\
\text { Amazon }\end{array}$ & present study \\
\hline- & - & D. giffardii Silvestri, 1913 & $?$ & De Santis 1979, pg. 68 \\
\hline- & - & D. kirbyi (Ashmead, 1904)* & $\begin{array}{l}\text { Peruvian } \\
\text { Amazon }\end{array}$ & present study \\
\hline Haltichellinae & - & - & - & - \\
\hline- & Haltichellini & - & - & - \\
\hline- & - & Aspirrhina bifurca Halstead, 1991* & $\begin{array}{l}\text { Peruvian } \\
\text { Amazon }\end{array}$ & present study \\
\hline- & - & A. dubitator (Walker, 1862)* & $\begin{array}{l}\text { Peruvian } \\
\text { Amazon }\end{array}$ & present study \\
\hline- & - & A. remotor (Walker, 1862)* & $\begin{array}{l}\text { Peruvian } \\
\text { Amazon }\end{array}$ & present study \\
\hline- & - & Ecuada producta Bouček, 1992 & $?$ & Bouček 1992, pg. 68 \\
\hline
\end{tabular}




\begin{tabular}{|c|c|c|c|c|}
\hline Subfamily & Tribe & scientificName & Location & references \\
\hline- & - & Haltichella spp.* & $\begin{array}{l}\text { Peruvian } \\
\text { Amazon }\end{array}$ & present study \\
\hline- & - & Hockeria sp.* & $\begin{array}{l}\text { Peruvian } \\
\text { Amazon }\end{array}$ & present study \\
\hline- & Hybothoracini & - & - & - \\
\hline- & - & Notaspidium acutum Halstead, 1991* & $\begin{array}{l}\text { Peruvian } \\
\text { Amazon }\end{array}$ & present study \\
\hline- & - & N. apantelis Bouček, 1992 & Pacific Coast & Bouček 1992, pg. 73 \\
\hline- & - & N. boharti Halstead, 1991* & $\begin{array}{l}\text { Peruvian } \\
\text { Amazon }\end{array}$ & present study \\
\hline- & - & N. braziliensis Halstead, 1991* & $\begin{array}{l}\text { Peruvian } \\
\text { Amazon }\end{array}$ & present study \\
\hline- & - & N. burdicki Halstead, 1991* & $\begin{array}{l}\text { Peruvian } \\
\text { Amazon }\end{array}$ & present study \\
\hline- & - & N. minutum Halstead, 1991* & $\begin{array}{l}\text { Peruvian } \\
\text { Amazon }\end{array}$ & present study \\
\hline- & - & N. truncatum Halstead, 1991* & $\begin{array}{l}\text { Peruvian } \\
\text { Amazon }\end{array}$ & present study \\
\hline- & & N. villegasi Halstead, 1991* & $\begin{array}{l}\text { Peruvian } \\
\text { Amazon }\end{array}$ & present study \\
\hline- & - & N. giganteum Halstead, 1991 * & $\begin{array}{l}\text { Peruvian } \\
\text { Amazon }\end{array}$ & Halstead 1991, pg. 233 \\
\hline- & - & Zavoya brevispina Bouček, 1992 & $\begin{array}{l}\text { Peruvian } \\
\text { Amazon }\end{array}$ & Bouček 1992, pg. 59 \\
\hline
\end{tabular}

With the rapid decline and loss of biodiversity, mainly caused by deforestation and land fragmentation (Chavez 2013, Asner et al. 2017), studying and describing the biodiversity of these areas at an accelerated pace has become a challenge for taxonomists (Myers et al. 2000, Erwin et al. 2007). Integrative taxonomy can be a useful tool for the discovery and documentation of biodiversity, by combining morphological characters and DNA barcoding approaches, providing accessible and applicable data to support conservation.

The present study is the result of a joint effort of the SNSB-Zoologische Staatssammlung München, Germany (ZSM) and the Insect Biodiversity Laboratory at Universidade Federal do Espírito Santo, Vitória, Brazil (LaBI-UFES) that aimed at providing an accelerated taxonomic study of the Chalcididae of the Panguana research station (Huanuco province) using traditional morphological approaches in combination with DNA barcoding. 


\section{Material and methods}

The specimens were collected between 2000 and 2017 using Malaise traps (Townes 1972) placed at irregular intervals (representing around 500 trap-days) at the Panguana reserve, Peru $\left(9.6137^{\circ} \mathrm{S}, 74.9356^{\circ} \mathrm{W}\right)$. Panguana is located at approximately $230 \mathrm{~m}$ elevation on the Yuyapichis river, occupying an area of 950 hectares (9.5 square kilometres) covered mainly by tropical primary forest, with an annual average temperature of $24.5^{\circ} \mathrm{C}$ and 2500 $\mathrm{mm}$ of precipitation distributed in about 180 days per year. Samples from sixteen Malaise traps from ten different years were sorted and Chalcididae extracted. Additional samples were obtained in December 2017 from canopy fogging (Floren 2010), resulting in 48 samples, of which eight samples contained specimens of Chalcididae that were included in our study.

\section{Workflow}

After the signature of a Memorandum of Understanding (MoU) between UFES and ZSM, a joint effort was settled by BCA, SS and MTT to accelerate the work process by using the ZSM expertise in molecular biology and LaBI-UFES expertise in Chalcididae taxonomy. BCA was responsible for the general coordination, samples sorting and genera identification (one week for sorting and identification), SS was in charge of the molecular coordination and MTT was in charge of the taxonomic coordination. MTT selected the specialists for each genus found during the sorting process. The specimens were illustrated following the specialist's request and the images sent via the ZSM server. Over one thousand images were prepared and sent in two weeks. Since Conura represented almost $40 \%$ of all specimens, TRAB was sent to ZSM to accelerate the identification process (one month). In order to avoid shipping a large amount of material, only a few specimens, not identified by the images, were sent from Germany to Brazil according to the specialist's request. Each specialist made the first identification of the material and selected the specimens with priority on the molecular pipeline. The material was plated in ZSM, sent to CCDB and the results analysed by SS, who communicated with the specialists and coordinated new rounds of molecular analysis. This process took around two months and was important to avoid sequencing the same species several times, thereby saving money and time. BCA, MTT and SS coordinated, together with the specialists, the interpretation of the morphological and molecular results and the definition of the species lists. The specialists prepared the results and discussion for each genus under MTT coordination (one month). BCA, MTT and PMS prepared the backbone of the manuscript while SS wrote the methodology. BCA, MTT and SS wrote the general discussion. In the end, all authors made changes and suggestions in the entire manuscript. All this coordination was performed by using e-mail and message apps, allowing real-time discussion. The entire process, from the beginning of the samples sorting until the paper submission, including delays, took ten months. 


\section{Taxonomic data assessment}

The taxonomic data compilation for the named specimens was based mainly on Noyes (2018). The data for each species is listed in Table 1.

\section{Morphological analysis}

Specimens were sorted to genus level at ZSM and then forwarded to taxonomic specialists at LaBI-UFES. Specimens for DNA barcoding were selected by the specialists based on morphological examination, aiming to obtain a set of specimens that reflected the species diversity. Long series of the same species were excluded to reduce cost and effort for the molecular analyses. Whenever possible, the specimens were identified to species level. Putative new species were flagged at this step. All specimens were imaged using a Nikon V1 camera attached to a Leica Z16 APO objective. Most specimens became part of revisional studies conducted as masters or doctoral theses at LaBI-UFES. The new species that were discovered will be formally described as part of the resulting taxonomic treatments of Neotropical and world generic revisions.

\section{DNA sequencing}

Whole specimens were submitted to the Canadian Centre for DNA Barcoding (CCDB) in Guelph, Canada, for DNA extraction and sequencing. The voucher specimens were submitted to non-destructive DNA extraction and sequencing for subsequent preparation and morphological study. DNA extraction, PCR amplification and sequencing were conducted at the CCDB using standardised protocols (Ivanova et al. 2006, deWaard et al. 2008, Ratnasingham and Hebert 2007 http://ccdb.ca/). The 658 bp target region, starting from the 5' end of the mitochondrial cytochrome c oxidase subunit I (COI) gene, includes the DNA barcode region for the animal kingdom (Hebert et al. 2003). The DNA extracts are stored at the CCDB. All specimen data are available through the Barcode of Life Database (BOLD) as a single, citable dataset (dx.doi.org/10.5883/DS-PECHAL1). The dataset includes collecting locality, geographic coordinates, elevation, collector, one or more digital images, identifier and voucher depository. Sequence data can be obtained through BOLD and include a detailed LIMS report, including chromatograms, primer information and access to trace files. The sequences are also available on GenBank (Accession nos. MK202011-MK202148).

Data on genetic material contained in this paper and the Barcode of Life Database (BOLD) are published for non-commercial use only, according to the agreements with the country providing the analysed samples. Use by third parties for purposes other than noncommercial scientific research may infringe the conditions under which the genetic resources were originally accessed and should not be undertaken without obtaining consent from the corresponding author of the paper and/or obtaining permission from the original providers of the genetic material. 


\section{Data analysis}

Sequences were aligned using the BOLD Aligner (amino acid-based hidden Markov models). The analyses are based on sequences with a minimum length of $300 \mathrm{bp}$ and < $1 \%$ ambiguous bases. Genetic distances and summary statistics were calculated using analytical tools in BOLD and are given as mean and maximum pairwise distances for intraspecific variation and as minimum pairwise distances for interspecific variations.

Sequence divergence statistics were calculated using the Kimura two-parameter model of sequence evolution (Kimura 1980). The Barcode Index Numbers (BINs) that are assigned by the BOLD system represent globally unique identifiers for clusters of sequences that correspond closely to putative species (Ratnasingham and Hebert 2013). For BIN assignment, a minimum sequence length of $500 \mathrm{bp}$ is required and sequences between 300 and 500 bp can join an existing BIN, but will not create or split BINs. BINs provide an interim taxonomic system and a way to determine Molecular Operational Taxonomic Units (MOTUs), prior to detailed taxonomic studies including morphology.

Sequences between 100 and 499 bp that did not meet the requirements for BIN assignment were submitted to a cluster analysis that included all sequences with a length of at least $100 \mathrm{bp}$. The analysis tool is provided by the BOLD system and generates OTUs independent of the BIN registry, using the REfined Single Linkage algorithm (RESL - Ratnasingham and Hebert 2013). In the tables presenting Sample IDs and BINs, "N/A" was used for missing data.

\section{Results}

A total of 418 specimens of Chalcididae were morphologically examined. The malaise traps provided 400 specimens and canopy fogging, the remaining 18 . The morphological analyses revealed 183 species of Chalcididae in ten genera (Fig. 1): 49 described species and at least 134 new species (Suppl. material 1). From a total of 76 described species found in the samples, only 10 species previously known to occur in Peru were recovered (Table 1), resulting in 66 new occurrence records for the country. Four genera (Aspirrhina, Haltichella, Hockeria and Melanosmicra) are also new distribution records for Peru, bringing the current total of Peruvian Chalcididae to 13 genera and 210 species (76 described and 134 new to science) (Suppl. material 1).

We submitted 268 of these 418 specimens representing 167 species to molecular analysis. Of those, 141 specimens yielded $\mathrm{COI}$ barcode region sequences, 136 of them with at least $300 \mathrm{bp}$. Sixty specimens were assigned a BIN by the BOLD system, resulting in 50 BINs. A cluster analysis with the 138 individuals that produced sequences longer than $100 \mathrm{bp}$ revealed 118 putative OTUs (putative species) with almost full congruence with the morphological analyses performed (Table 2). 


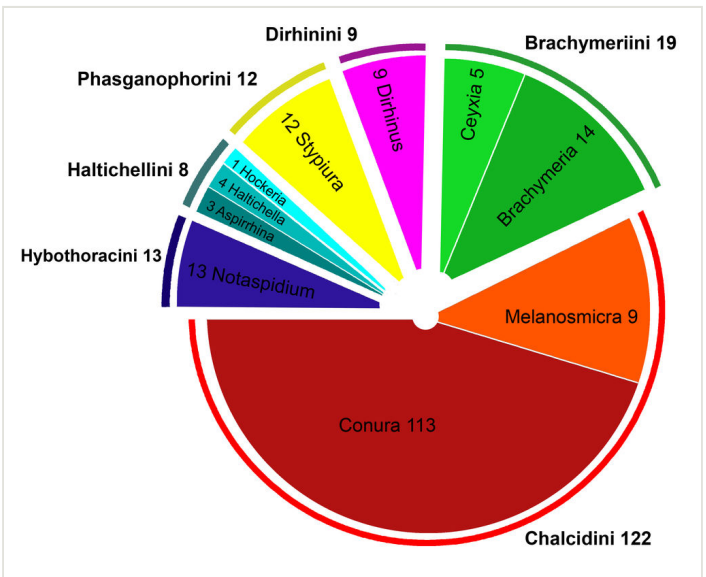

Figure 1. doi

Proportion of Chalcididae species recovered in samples from Panguana station, Huánuco, Peruvian Amazon, per genera and tribes.

Table 2.

Cluster analysis of sequences obtained from 138 specimens, with minimum length of $100 \mathrm{bp}$ (BIN: Barcode Index Number; NN Dist: distance to the nearest neighbour).

\begin{tabular}{|c|c|c|c|c|c|}
\hline OTU & materialSampleID & Genus & Species & BIN & NN Dist \\
\hline OTU-1 & BC-ZSM-HYM-30556-H10 & Brachymeria & sp11 & N/A & 16.031 \\
\hline OTU-2 & BC-ZSM-HYM-27511-F04 & Brachymeria & sp5 & ADF1042 & 10.417 \\
\hline OTU-3 & BC-ZSM-HYM-27511-F05 & Brachymeria & sp10 & ADF1766 & 10.417 \\
\hline OTU-3 & BC-ZSM-HYM-30556-H06 & Brachymeria & sp10 & ADF1766 & 10.417 \\
\hline OTU-3 & BC-ZSM-HYM-30556-H07 & Brachymeria & sp10 & ADF1766 & 10.417 \\
\hline OTU-4 & BC-ZSM-HYM-25927-G12 & Brachymeria & $\mathrm{sp} 4$ & ADH9819 & 14.626 \\
\hline OTU-5 & BC-ZSM-HYM-25927-G11 & Brachymeria & $\mathrm{sp} 7$ & $\mathrm{~N} / \mathrm{A}$ & 10.549 \\
\hline OTU-6 & BC-ZSM-HYM-25927-G05 & Brachymeria & sp3 & ADI0920 & 10.549 \\
\hline OTU-6 & BC-ZSM-HYM-30556-H08 & Brachymeria & sp3 & ADI0920 & 10.549 \\
\hline OUT-7 & BC-ZSM-HYM-30556-B05 & Brachymeria & sp6 & $\mathrm{N} / \mathrm{A}$ & 9.343 \\
\hline OUT-8 & BC-ZSM-HYM-30556-B08 & Brachymeria & pandora & $\mathrm{N} / \mathrm{A}$ & 9.343 \\
\hline OUT-8 & BC-ZSM-HYM-30556-B09 & Brachymeria & pandora & N/A & 9.343 \\
\hline OUT-9 & BC-ZSM-HYM-30556-B04 & Ceyxia & sp1 & $\mathrm{N} / \mathrm{A}$ & 12.121 \\
\hline OTU-10 & BC-ZSM-HYM-30556-F02 & Conura & sp62 & $\mathrm{N} / \mathrm{A}$ & 9.848 \\
\hline OTU-11 & BC-ZSM-HYM-30556-F05 & Conura & sp65 & $\mathrm{N} / \mathrm{A}$ & 7.323 \\
\hline
\end{tabular}




\begin{tabular}{|c|c|c|c|c|c|}
\hline OTU & materialSampleID & Genus & Species & BIN & NN Dist \\
\hline OTU-12 & BC-ZSM-HYM-30556-E11 & Conura & sp59 & $N / A$ & 6.818 \\
\hline OTU-13 & BC-ZSM-HYM-30556-E12 & Conura & sp60 & $\mathrm{N} / \mathrm{A}$ & 9.848 \\
\hline OTU-14 & BC-ZSM-HYM-30556-G05 & Conura & sp78 & ADO4114 & 78.283 \\
\hline OTU-15 & BC-ZSM-HYM-30556-G06 & Conura & sp79 & $\mathrm{N} / \mathrm{A}$ & 10.354 \\
\hline OTU-16 & BC-ZSM-HYM-30556-G03 & Conura & sp75 & $\mathrm{N} / \mathrm{A}$ & 7.323 \\
\hline OTU-17 & BC-ZSM-HYM-30556-G04 & Conura & sp77 & $\mathrm{N} / \mathrm{A}$ & 10.744 \\
\hline OTU-18 & BC-ZSM-HYM-30556-G01 & Conura & sp73 & $\mathrm{N} / \mathrm{A}$ & 6.061 \\
\hline OTU-19 & BC-ZSM-HYM-30556-G02 & Conura & sp74 & $\mathrm{N} / \mathrm{A}$ & 8.081 \\
\hline OTU-20 & BC-ZSM-HYM-30556-F11 & Conura & sp71 & $\mathrm{N} / \mathrm{A}$ & 7.576 \\
\hline OTU-21 & BC-ZSM-HYM-30556-F12 & Conura & sp72 & $\mathrm{N} / \mathrm{A}$ & 9.091 \\
\hline OTU-22 & BC-ZSM-HYM-30556-H05 & Conura & femorata & $\mathrm{N} / \mathrm{A}$ & 10.499 \\
\hline OTU-23 & BC-ZSM-HYM-30556-H03 & Conura & ferruginea & $\mathrm{N} / \mathrm{A}$ & 8.192 \\
\hline OTU-24 & BC-ZSM-HYM-30556-H01 & Conura & sp84 & $\mathrm{N} / \mathrm{A}$ & 8.267 \\
\hline OTU-25 & BC-ZSM-HYM-30556-G10 & Conura & sp81 & $\mathrm{N} / \mathrm{A}$ & 11.616 \\
\hline OTU-26 & BC-ZSM-HYM-30556-G09 & Conura & chapadae & $\mathrm{N} / \mathrm{A}$ & 8.267 \\
\hline OTU-27 & BC-ZSM-HYM-30556-G07 & Conura & sp80 & $\mathrm{N} / \mathrm{A}$ & 5.823 \\
\hline OTU-28 & BC-ZSM-HYM-27511-F11 & Conura & sp15 & ADE9828 & 8.208 \\
\hline OTU-29 & BC-ZSM-HYM-27511-G02 & Conura & sp42 & $\mathrm{N} / \mathrm{A}$ & 9.283 \\
\hline OTU-30 & BC-ZSM-HYM-27511-F12 & Conura & sp12 & ADE9959 & 5.800 \\
\hline OTU-31 & BC-ZSM-HYM-27511-G05 & Conura & sp13 & ADE9831 & 74.074 \\
\hline OTU-32 & BC-ZSM-HYM-27511-G04 & Conura & sp16 & ADE9956 & 79.545 \\
\hline OTU-33 & BC-ZSM-HYM-27511-G07 & Conura & sp03 & ADE9827 & 23.173 \\
\hline OTU-34 & BC-ZSM-HYM-27511-G06 & Conura & sp02 & ADE9823 & 9.717 \\
\hline OTU-34 & BC-ZSM-HYM-25927-C05 & Conura & sp02 & ADE9823 & 9.717 \\
\hline OTU-34 & BC-ZSM-HYM-25927-D04 & Conura & sp02 & ADE9823 & 9.717 \\
\hline OTU-35 & BC-ZSM-HYM-27511-G09 & Conura & sp17 & ADE9958 & 9.319 \\
\hline OTU-36 & BC-ZSM-HYM-27511-G08 & Conura & sp03 & ADE9681 & 23.173 \\
\hline OTU-36 & BC-ZSM-HYM-27511-H11 & Conura & sp03 & ADE9681 & 23.173 \\
\hline OTU-37 & BC-ZSM-HYM-27511-G11 & Conura & sp19 & ADE9960 & 9.259 \\
\hline OTU-38 & BC-ZSM-HYM-27511-G10 & Conura & sp18 & ADE9682 & 9.667 \\
\hline
\end{tabular}




\begin{tabular}{|c|c|c|c|c|c|}
\hline OTU & materialSampleID & Genus & Species & BIN & NN Dist \\
\hline OTU-39 & BC-ZSM-HYM-27511-H02 & Conura & nigrifrons & ADE9829 & 74.074 \\
\hline OTU-40 & BC-ZSM-HYM-27511-G12 & Conura & sp20 & ADE9957 & 7.317 \\
\hline OTU-41 & BC-ZSM-HYM-27511-H04 & Conura & sp90 & ADE9826 & 5.800 \\
\hline OTU-42 & BC-ZSM-HYM-27511-H03 & Conura & sp18 & ADE9822 & 9.667 \\
\hline OTU-43 & BC-ZSM-HYM-27511-H07 & Conura & sp22 & ADE9825 & 8.333 \\
\hline OTU-44 & BC-ZSM-HYM-27511-H08 & Conura & debilis & ADE9824 & 11.787 \\
\hline OTU-45 & BC-ZSM-HYM-27511-H05 & Conura & sp21 & ADE9830 & 11.295 \\
\hline OTU-46 & BC-ZSM-HYM-27511-H06 & Conura & sp06 & ADE9680 & 25.126 \\
\hline OTU-47 & BC-ZSM-HYM-25927-A07 & Conura & sp04 & ADI0628 & 10.452 \\
\hline OTU-47 & BC-ZSM-HYM-25927-B01 & Conura & sp04 & ADI0628 & 10.452 \\
\hline OTU-48 & BC-ZSM-HYM-25927-A08 & Conura & sp05 & ADI0820 & 8.333 \\
\hline OTU-49 & BC-ZSM-HYM-25927-A02 & Conura & dares & $\mathrm{N} / \mathrm{A}$ & 6.790 \\
\hline OTU-50 & BC-ZSM-HYM-25927-A04 & Conura & sp23 & $\mathrm{N} / \mathrm{A}$ & 12.393 \\
\hline OTU-51 & BC-ZSM-HYM-25927-A11 & Conura & sp07 & ADI1177 & 11.111 \\
\hline OTU-52 & BC-ZSM-HYM-25927-B02 & Conura & sp01 & ADI0629 & 9.848 \\
\hline OTU-53 & BC-ZSM-HYM-25927-A09 & Conura & camescens & ADI0819 & 8.267 \\
\hline OTU-54 & BC-ZSM-HYM-25927-A10 & Conura & sp06 & ADI0818 & 25.126 \\
\hline OTU-55 & BC-ZSM-HYM-25927-B11 & Conura & sp30 & $\mathrm{N} / \mathrm{A}$ & 10.840 \\
\hline OTU-56 & BC-ZSM-HYM-25927-C01 & Conura & sp09 & ADI0821 & 2.322 \\
\hline OTU-57 & BC-ZSM-HYM-25927-B06 & Conura & amoena & ADI0816 & 12.169 \\
\hline OTU-58 & BC-ZSM-HYM-25927-B10 & Conura & sp08 & ADI0627 & 8.202 \\
\hline OTU-59 & BC-ZSM-HYM-25927-C06 & Conura & pygmaea & ADI0817 & 8.757 \\
\hline OTU-60 & BC-ZSM-HYM-25927-C04 & Conura & sp10 & ADI1239 & 6.933 \\
\hline OTU-61 & BC-ZSM-HYM-25927-C03 & Conura & sp89 & ADH8745 & 2.322 \\
\hline OTU-62 & BC-ZSM-HYM-25927-C02 & Conura & adela & ADI1049 & 8.429 \\
\hline OTU-63 & BC-ZSM-HYM-25927-C09 & Conura & sp11 & ADI0626 & 8.800 \\
\hline OTU-64 & BC-ZSM-HYM-25927-C07 & Conura & sp32 & $\mathrm{N} / \mathrm{A}$ & 6.962 \\
\hline OTU-65 & BC-ZSM-HYM-25927-H09 & Conura & sp14 & ADI0822 & 9.649 \\
\hline OTU-66 & BC-ZSM-HYM-30556-D09 & Conura & mourei & $\mathrm{N} / \mathrm{A}$ & 1.032 \\
\hline OTU-67 & BC-ZSM-HYM-30556-D08 & Conura & sp47 & $\mathrm{N} / \mathrm{A}$ & 9.366 \\
\hline
\end{tabular}




\begin{tabular}{|c|c|c|c|c|c|}
\hline OTU & materialSampleID & Genus & Species & BIN & NN Dist \\
\hline OTU-68 & BC-ZSM-HYM-30556-D11 & Conura & sp49 & $N / A$ & 11.111 \\
\hline OTU-69 & BC-ZSM-HYM-30556-D10 & Conura & sp48 & $\mathrm{N} / \mathrm{A}$ & 7.323 \\
\hline OTU-70 & BC-ZSM-HYM-30556-D02 & Conura & sp35 & $\mathrm{N} / \mathrm{A}$ & 7.576 \\
\hline OTU-70 & BC-ZSM-HYM-30556-H02 & Conura & immaculata & $\mathrm{N} / \mathrm{A}$ & 7.576 \\
\hline OTU-71 & BC-ZSM-HYM-30556-D07 & Conura & sp46 & $\mathrm{N} / \mathrm{A}$ & 6.061 \\
\hline OTU-72 & BC-ZSM-HYM-30556-D05 & Conura & vesicula & $\mathrm{N} / \mathrm{A}$ & 8.642 \\
\hline OTU-73 & BC-ZSM-HYM-30556-E08 & Conura & sp57 & $\mathrm{N} / \mathrm{A}$ & 7.163 \\
\hline OTU-74 & BC-ZSM-HYM-30556-E07 & Conura & sp56 & $\mathrm{N} / \mathrm{A}$ & 78.283 \\
\hline OTU-75 & BC-ZSM-HYM-30556-E10 & Conura & bidentata & N/A & 8.800 \\
\hline OTU-76 & BC-ZSM-HYM-30556-E09 & Conura & sp58 & $\mathrm{N} / \mathrm{A}$ & 6.313 \\
\hline OTU-77 & BC-ZSM-HYM-30556-E03 & Conura & dorsimaculata & $\mathrm{N} / \mathrm{A}$ & 6.790 \\
\hline OTU-78 & BC-ZSM-HYM-30556-D12 & Conura & sp50 & $\mathrm{N} / \mathrm{A}$ & 6.061 \\
\hline OTU-79 & BC-ZSM-HYM-30556-E06 & Conura & sp55 & $\mathrm{N} / \mathrm{A}$ & 9.091 \\
\hline OTU-80 & BC-ZSM-HYM-30556-E04 & Conura & sp53 & $\mathrm{N} / \mathrm{A}$ & 5.823 \\
\hline OTU-81 & BC-ZSM-HYM-30556-F09 & Conura & sp69 & $\mathrm{N} / \mathrm{A}$ & 8.000 \\
\hline OTU-82 & BC-ZSM-HYM-30556-F10 & Conura & sp70 & $\mathrm{N} / \mathrm{A}$ & 6.061 \\
\hline OTU-83 & BC-ZSM-HYM-30556-F07 & Conura & sp67 & $\mathrm{N} / \mathrm{A}$ & 7.323 \\
\hline OTU-84 & BC-ZSM-HYM-30556-F08 & Conura & sp68 & $\mathrm{N} / \mathrm{A}$ & 9.409 \\
\hline OUT-85 & BC-ZSM-HYM-30556-C03 & Conura & sp91 & ADO1707 & 1.009 \\
\hline OUT-86 & BC-ZSM-HYM-27511-C03 & Dirhinus & buscki & ADF1234 & 28.620 \\
\hline OUT-86 & BC-ZSM-HYM-27511-C09 & Dirhinus & buscki & ADF1234 & 28.620 \\
\hline OUT-86 & BC-ZSM-HYM-27511-C11 & Dirhinus & buscki & ADF1234 & 28.620 \\
\hline OUT-86 & BC-ZSM-HYM-25927-E04 & Dirhinus & buscki & ADF1234 & 28.620 \\
\hline OTU-87 & BC-ZSM-HYM-25927-E03 & Dirhinus & sp1 & $\mathrm{N} / \mathrm{A}$ & 8.000 \\
\hline OTU-88 & BC-ZSM-HYM-27511-C04 & Dirhinus & kirbyi & ADE9658 & 8.065 \\
\hline OTU-88 & BC-ZSM-HYM-27511-C10 & Dirhinus & kirbyi & ADE9658 & 8.065 \\
\hline OTU-89 & BC-ZSM-HYM-30556-B01 & Dirhinus & cameroni & $\mathrm{N} / \mathrm{A}$ & 15.301 \\
\hline OTU-90 & BC-ZSM-HYM-30556-B03 & Dirhinus & $\mathrm{sp} 4$ & $\mathrm{~N} / \mathrm{A}$ & 8.065 \\
\hline OTU-91 & BC-ZSM-HYM-27511-C06 & Dirhinus & sp6 & ADE9797 & 28.620 \\
\hline OTU-92 & BC-ZSM-HYM-30556-A03 & Haltichella & sp4 & $\mathrm{N} / \mathrm{A}$ & 13.889 \\
\hline
\end{tabular}




\begin{tabular}{|c|c|c|c|c|c|}
\hline отU & materialSampleID & Genus & Species & BIN & NN Dist \\
\hline OTU-93 & BC-ZSM-HYM-30556-A02 & Haltichella & sp3 & $N / A$ & 15.051 \\
\hline OTU-94 & BC-ZSM-HYM-27511-B01 & Melanosmicra & immaculata & ADF0815 & 53.333 \\
\hline OTU-94 & BC-ZSM-HYM-27511-B06 & Melanosmicra & immaculata & ADF0815 & 53.333 \\
\hline OTU-94 & BC-ZSM-HYM-27511-C01 & Melanosmicra & immaculata & ADF0815 & 53.333 \\
\hline OTU-94 & BC-ZSM-HYM-30556-C06 & Melanosmicra & immaculata & $N / A$ & 53.333 \\
\hline OTU-95 & BC-ZSM-HYM-27511-B02 & Melanosmicra & sp3 & ADF1337 & 22.727 \\
\hline OTU-95 & BC-ZSM-HYM-30556-C01 & Melanosmicra & sp3 & ADF1337 & 22.727 \\
\hline OTU-96 & BC-ZSM-HYM-27511-B03 & Melanosmicra & sp1 & ADF1769 & 48.387 \\
\hline OTU-97 & BC-ZSM-HYM-27511-B11 & Melanosmicra & flavicolis & ADE9942 & 10.840 \\
\hline OTU-99 & BC-ZSM-HYM-30556-C05 & Melanosmicra & carenata & $N / A$ & 8.586 \\
\hline OTU-100 & BC-ZSM-HYM-30556-C08 & Melanosmicra & rugosa & $N / A$ & 11.295 \\
\hline OTU-100 & BC-ZSM-HYM-30556-C09 & Melanosmicra & rugosa & $\mathrm{N} / \mathrm{A}$ & 11.295 \\
\hline OTU-101 & BC-ZSM-HYM-30556-B12 & Melanosmicra & nigra & $\mathrm{N} / \mathrm{A}$ & 22.727 \\
\hline OTU-101 & BC-ZSM-HYM-30556-C07 & Melanosmicra & nigra & $\mathrm{N} / \mathrm{A}$ & 22.727 \\
\hline OTU-102 & BC-ZSM-HYM-30556-C02 & Melanosmicra & tricolor & $\mathrm{N} / \mathrm{A}$ & 48.387 \\
\hline OTU-102 & BC-ZSM-HYM-30556-C04 & Melanosmicra & tricolor & $\mathrm{N} / \mathrm{A}$ & 48.387 \\
\hline OTU-103 & BC-ZSM-HYM-27511-E03 & Notaspidium & minutum & ADE9953 & 13.115 \\
\hline OTU-104 & BC-ZSM-HYM-25927-E08 & Notaspidium & truncatum & $\mathrm{N} / \mathrm{A}$ & 11.852 \\
\hline OTU-105 & BC-ZSM-HYM-25927-E09 & Notaspidium & $\mathrm{sp} 2$ & ADJ2148 & 1.016 \\
\hline OTU-106 & BC-ZSM-HYM-30556-A06 & Notaspidium & sp1 & N/A & 13.333 \\
\hline OTU-107 & BC-ZSM-HYM-30556-A12 & Notaspidium & sp4 & $N / A$ & 10.298 \\
\hline OTU-108 & BC-ZSM-HYM-30556-A07 & Notaspidium & apantelis & N/A & 1.221 \\
\hline OTU-109 & BC-ZSM-HYM-30556-A08 & Notaspidium & braziliensis & $\mathrm{N} / \mathrm{A}$ & 1.221 \\
\hline OTU-110 & BC-ZSM-HYM-30556-A10 & Notaspidium & burdicki & N/A & 83.951 \\
\hline OTU-111 & BC-ZSM-HYM-30556-A11 & Notaspidium & sp5 & $\mathrm{N} / \mathrm{A}$ & 83.951 \\
\hline OTU-112 & BC-ZSM-HYM-27511-D10 & Notaspidium & acutum & ADF1074 & 1.368 \\
\hline OTU-113 & BC-ZSM-HYM-27511-D11 & Notaspidium & boharti & ADF1075 & 10.298 \\
\hline OTU-114 & BC-ZSM-HYM-27511-E07 & Stypiura & $\mathrm{sp} 2$ & $\mathrm{~N} / \mathrm{A}$ & 58.952 \\
\hline OTU-115 & BC-ZSM-HYM-27511-E12 & Stypiura & sp7 & $\mathrm{N} / \mathrm{A}$ & 8.955 \\
\hline OTU-116 & BC-ZSM-HYM-27511-F02 & Stypiura & sp6 & ADF0812 & 58.952 \\
\hline
\end{tabular}




\begin{tabular}{|l|l|l|l|l|l|}
\hline OTU & materialSampleID & Genus & Species & BIN & NN Dist \\
\hline OTU-117 & BC-ZSM-HYM-27511-F03 & Stypiura & batesii & N/A & 69.565 \\
\hline OTU-118 & BC-ZSM-HYM-30556-C12 & Stypiura & sp8 & N/A & 8.036 \\
\hline
\end{tabular}

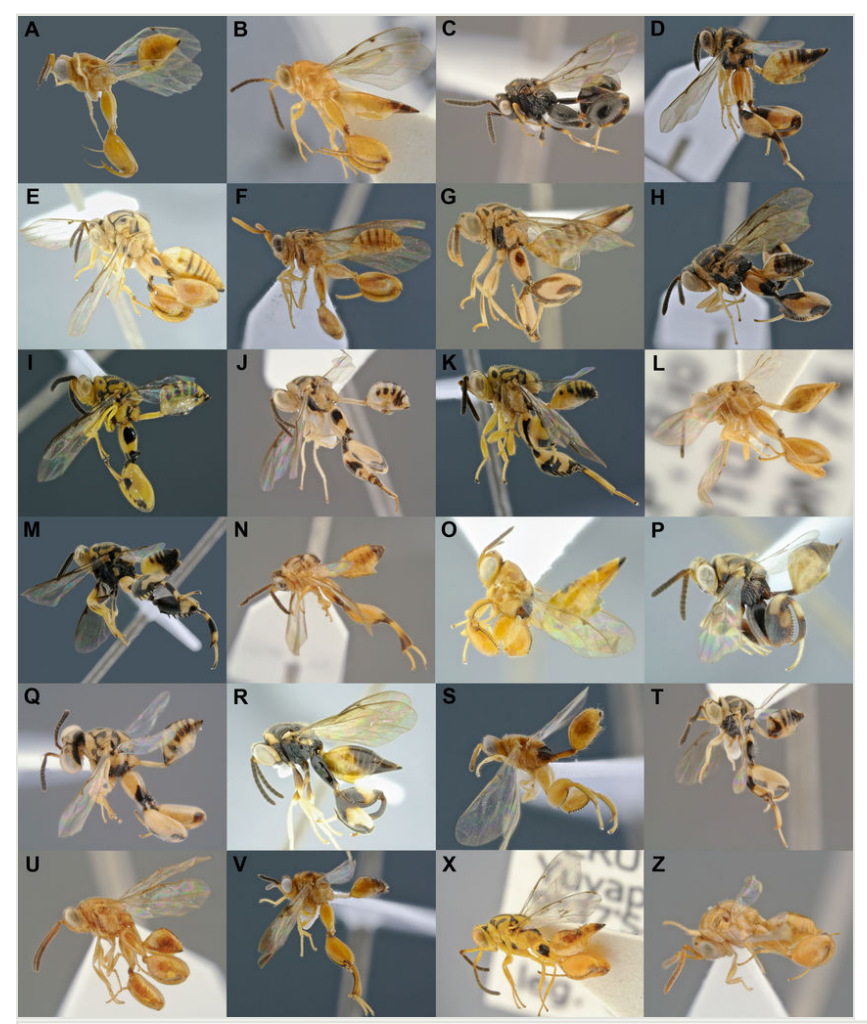

Figure 2. doi

Diversity of Conura subgenera and species groups (Delvare 1992) and respective BINs. All specimens are females, except F and K: A. Co. (Ceratosmicra) camescens (immaculata group; BOLD:ADI0819); B. Co. (Ce.) sp77 (onorei group); C. Co. (Ce.) dorsimaculata (side group); D. Co. (Conura) dares (dares group); E. Co. (Co.) nigrifrons (maculata group; BOL D:ADE9829); F. Co. (Co.) sp90 (vau group); G. Co. (Spilochalcis) sp76 (picta group); H. Co. (Sp.) sp04 (chrysomera group; BOLD:ADI0628); I. Co. (Sp.) sp23 (surumuae group); J. Co. (Sp.) vesicula (vesicula group); K. Co. (Sp.) sp29 (annulipes group); L. Co. (Sp.) sp45 (femorata group); M. Co. (Sp.) amoena (flava group; BOLD:ADI0816); N. Co. (Sp.) sp63 (pygmaea group); O. Co. (Sp.) sp16 (aequalis group; BOLD:ADE9956); P. Co. (Sp.) sp17 (arcuaspina group; BOLD:ADE9958); Q. Co. (Sp.) sp68 (contributa group); R. Co. (Sp.) santaremensis (discolor group); S. Co. (Sp.) adela (maculipennis group; BOLD:ADI10 49); T. Co. (Sp.) sp62 (rasplusi group); U. Co. (Sp.) sp44 (rufodorsalis group); V. Co. (Sp.) sp89 (xanthostigma group; BOLD:ADH8745) ; X. Co. (Sp.) attacta (femorata group); Z. Co. (Ce.) immaculata (immaculata group). 


\section{Conura Spinola, 1837}

Conura was the most abundant and richest genus sampled with a total of 166 specimens sorted into 113 species, a trend found in other checklist papers of Chalcididae (Arias and Delvare 2003, Tavares and de Araujo 2007). It was the second most common and diverse genus in the fogging samples, with six specimens representing six species, (one formally described and five new species). Conura currently includes 301 valid species (Noyes 2018), with 8 recorded in Peru so far (Table 1). This study recovered two of those species, Co. attacta and Co. immaculata and added another 22, totalling 30 described species for the country (Fig. 2). At least 37 species obtained in this study are new species, which are being described in papers in preparation at LaBI-UFES. One species (Co. sp90) was represented by 11 specimens and another two (Co. sp3 and Co. sp35) by six specimens; however, $77 \%$ of the sampled species were singletons. Despite being the second most dominant genus in the fogging samples, only six species singletons were obtained.

Conura systematics is rather complicated, with three subgenera, three species complexes and 63 species groups (Delvare 1992). All subgenera and species complexes were represented in the samples, as well as 24 of the 63 species groups (Suppl. material 1). A total of 135 specimens, representing the majority of sampled species of Conura, were chosen for the molecular pipeline. Of those, 80 specimens produced sequences of at least $200 \mathrm{bp}$ and were included in the cluster analysis (Table 3). The clusters presented an average of $13.51 \%$ nearest neighbour (NN) distance for the genus Conura. Comparing the subgenera separately, the average nearest neighbour (NN) distance is $8.32 \%$ for the subgenus Ceratosmicra, $24.63 \%$ for subgenus Conura and $12.48 \%$ for subgenus Spilochalcis. Thirty-seven BINs were associated with the specimens sequenced, all new to BOLD (Table 3).

\section{Table 3.}

Conura specimens, corresponding BINs and BOLD data of the specimens submitted to the molecular pipeline producing sequences longer than $200 \mathrm{bp}$.

\begin{tabular}{|l|l|l|l|}
\hline scientificName & Specimens & BIN & materialSampleID \\
\hline Co. adela & 1 & ADI1049 & BC-ZSM-HYM-25927-C02 \\
\hline Co. amoena & 1 & ADI0816 & BC-ZSM-HYM-25927-B06 \\
\hline Co. bidentata & 1 & N/A & BC-ZSM-HYM-30556-E10 \\
\hline Co. camenscens & 1 & ADI0819 & BC-ZSM-HYM-25927-A09 \\
\hline Co. chapadae & 1 & N/A & BC-ZSM-HYM-30556-G09 \\
\hline Co. dares & 2 & N/A & BC-ZSM-HYM-25927-A02 \\
\hline Co. debilis & 1 & ADE9824 & BC-ZSM-HYM-27511-H08 \\
\hline Co. dorsimaculata & 1 & N/A & BC-ZSM-HYM-30556-E03 \\
\hline
\end{tabular}




\begin{tabular}{|c|c|c|c|}
\hline scientificName & Specimens & BIN & materialSampleID \\
\hline Co. femorata & 1 & $\mathrm{~N} / \mathrm{A}$ & BC-ZSM-HYM-30556-H05 \\
\hline Co. ferruginea & 1 & N/A & BC-ZSM-HYM-30556-H03 \\
\hline Co. immaculata & 1 & N/A & BC-ZSM-HYM-30556-H02 \\
\hline Co. mourei & 1 & N/A & BC-ZSM-HYM-30556-D09 \\
\hline Co. nigrifrons & 1 & ADE9829 & BC-ZSM-HYM-27511-H02 \\
\hline Co. pygmaea & 1 & ADI0817 & BC-ZSM-HYM-25927-C06 \\
\hline Co. vesicula & 1 & N/A & BC-ZSM-HYM-30556-D05 \\
\hline Co. sp01 & 1 & ADI0629 & BC-ZSM-HYM-25927-B02 \\
\hline Co. sp02 & 3 & ADE9823 & $\begin{array}{l}\text { BC-ZSM-HYM-25927-C05; BC-ZSM-HYM-25927-D04; BC-ZSM- } \\
\text { HYM-27511-G06 }\end{array}$ \\
\hline Co. sp03 & 6 & $\begin{array}{l}\text { ADE9827; } \\
\text { ADE9681 }\end{array}$ & $\begin{array}{l}\text { BC-ZSM-HYM-27511-G07; BC-ZSM-HYM-27511-G08; BC-ZSM- } \\
\text { HYM-27511-H11 }\end{array}$ \\
\hline Co. sp04 & 2 & ADI0628 & BC-ZSM-HYM-25927-A07; BC-ZSM-HYM-25927-B01 \\
\hline Co. sp05 & 1 & ADI0820 & BC-ZSM-HYM-25927-A08 \\
\hline Co. sp06 & 2 & $\begin{array}{l}\text { ADI0818; } \\
\text { ADE9680 }\end{array}$ & BC-ZSM-HYM-25927-A10; BC-ZSM-HYM-27511-H06 \\
\hline Co. sp07 & 1 & ADI1177 & BC-ZSM-HYM-25927-A11 \\
\hline Co. sp08 & 1 & ADI0627 & BC-ZSM-HYM-25927-B10 \\
\hline Co. sp09 & 1 & ADI0821 & BC-ZSM-HYM-25927-C01 \\
\hline Co. sp10 & 1 & ADI1239 & BC-ZSM-HYM-25927-C04 \\
\hline Co. sp11 & 1 & ADI0626 & BC-ZSM-HYM-25927-C09 \\
\hline Co. sp12 & 3 & ADE9959 & BC-ZSM-HYM-27511-F12 \\
\hline Co. sp13 & 3 & ADE9831 & BC-ZSM-HYM-27511-G05 \\
\hline Co. sp14 & 1 & ADI0822 & BC-ZSM-HYM-25927-H09 \\
\hline Co. sp15 & 1 & ADE9828 & BC-ZSM-HYM-27511-F11 \\
\hline Co. sp16 & 1 & ADE9956 & BC-ZSM-HYM-27511-G04 \\
\hline Co. sp17 & 1 & ADE9958 & BC-ZSM-HYM-27511-G09 \\
\hline Co. sp18 & 2 & $\begin{array}{l}\text { ADE9682; } \\
\text { ADE9822 }\end{array}$ & BC-ZSM-HYM-27511-G10; BC-ZSM-HYM-27511-H03 \\
\hline Co. sp19 & 1 & ADE9960 & BC-ZSM-HYM-27511-G11 \\
\hline Co. sp20 & 1 & ADE9957 & BC-ZSM-HYM-27511-G12 \\
\hline
\end{tabular}




\begin{tabular}{|c|c|c|c|}
\hline scientificName & Specimens & BIN & materiaISampleID \\
\hline Co. sp21 & 1 & ADE9830 & BC-ZSM-HYM-27511-H05 \\
\hline Co. sp22 & 1 & ADE9825 & BC-ZSM-HYM-27511-H07 \\
\hline Co. sp23 & 1 & N/A & BC-ZSM-HYM-25927-A04 \\
\hline Co. sp30 & 1 & N/A & BC-ZSM-HYM-25927-B11 \\
\hline Co. sp32 & 1 & N/A & BC-ZSM-HYM-25927-C07 \\
\hline Co. sp35 & 7 & $\mathrm{~N} / \mathrm{A}$ & BC-ZSM-HYM-30556-D02 \\
\hline C. sp42 & 1 & N/A & BC-ZSM-HYM-27511-G02 \\
\hline Co. sp46 & 1 & $\mathrm{~N} / \mathrm{A}$ & BC-ZSM-HYM-30556-D07 \\
\hline Co. sp47 & 1 & $N / A$ & BC-ZSM-HYM-30556-D08 \\
\hline Co. sp48 & 3 & N/A & BC-ZSM-HYM-30556-D10 \\
\hline Co. sp49 & 3 & $N / A$ & BC-ZSM-HYM-30556-D11 \\
\hline Co. sp50 & 1 & N/A & BC-ZSM-HYM-30556-D12 \\
\hline Co. sp53 & 1 & $N / A$ & BC-ZSM-HYM-30556-E04 \\
\hline Co. sp55 & 1 & $\mathrm{~N} / \mathrm{A}$ & BC-ZSM-HYM-30556-E06 \\
\hline Co. sp56 & 1 & $N / A$ & BC-ZSM-HYM-30556-E07 \\
\hline Co. sp57 & 1 & N/A & BC-ZSM-HYM-30556-E08 \\
\hline Co. sp58 & 1 & N/A & BC-ZSM-HYM-30556-E09 \\
\hline Co. sp59 & 1 & N/A & BC-ZSM-HYM-30556-E11 \\
\hline Co. sp60 & 1 & N/A & BC-ZSM-HYM-30556-E12 \\
\hline Co. sp62 & 1 & $N / A$ & BC-ZSM-HYM-30556-F02 \\
\hline Co. sp65 & 1 & $N / A$ & BC-ZSM-HYM-30556-F05 \\
\hline Co. sp67 & 1 & N/A & BC-ZSM-HYM-30556-F07 \\
\hline Co. sp68 & 1 & $N / A$ & BC-ZSM-HYM-30556-F08 \\
\hline Co. sp69 & 1 & $N / A$ & BC-ZSM-HYM-30556-F09 \\
\hline Co. sp70 & 1 & $N / A$ & BC-ZSM-HYM-30556-F10 \\
\hline Co. sp71 & 1 & $\mathrm{~N} / \mathrm{A}$ & BC-ZSM-HYM-30556-F11 \\
\hline Co. sp72 & 1 & $\mathrm{~N} / \mathrm{A}$ & BC-ZSM-HYM-30556-F12 \\
\hline Co. sp73 & 1 & $\mathrm{~N} / \mathrm{A}$ & BC-ZSM-HYM-30556-G01 \\
\hline Co. sp74 & 1 & $\mathrm{~N} / \mathrm{A}$ & BC-ZSM-HYM-30556-G02 \\
\hline Co. sp75 & 1 & $\mathrm{~N} / \mathrm{A}$ & BC-ZSM-HYM-30556-G03 \\
\hline
\end{tabular}




\begin{tabular}{|l|l|l|l|}
\hline scientificName & Specimens & BIN & materialSampleID \\
\hline Co. sp77 & 1 & N/A & BC-ZSM-HYM-30556-G04 \\
\hline Co. sp78 & 1 & N/A & BC-ZSM-HYM-30556-G05 \\
\hline C. sp79 & 1 & N/A & BC-ZSM-HYM-30556-G06 \\
\hline Co. sp80 & 1 & N/A & BC-ZSM-HYM-30556-G07 \\
\hline Co. sp81 & 1 & N/A & BC-ZSM-HYM-30556-G10 \\
\hline Co. sp84 & 1 & N/A & BC-ZSM-HYM-30556-H01 \\
\hline Co. sp89 & 2 & ADH8745 & BC-ZSM-HYM-25927-C03 \\
\hline Co. sp90 & 8 & ADE9826 & BC-ZSM-HYM-27511-H04 \\
\hline
\end{tabular}

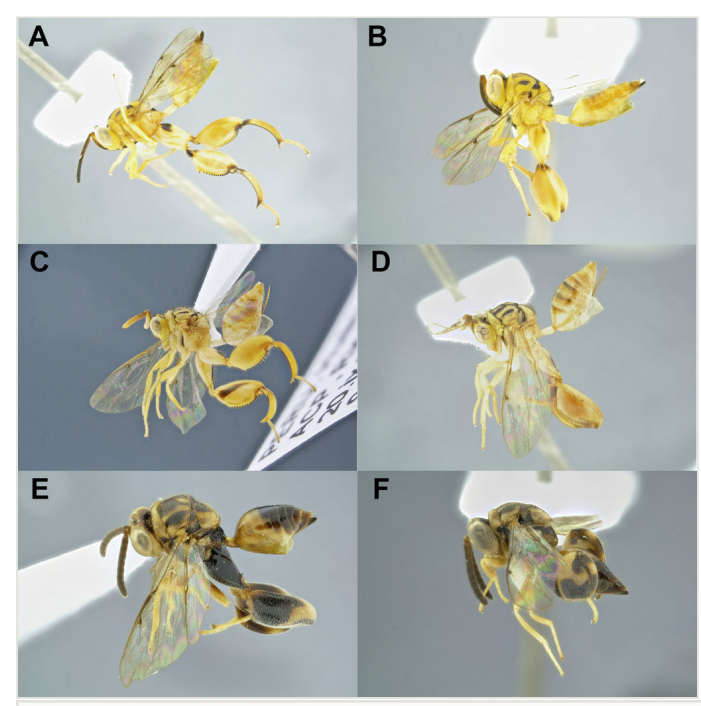

Figure 3. doi

Species of Conura with morphologically indistinct specimens and two BINs, females: $\mathbf{A}$ and B. Conura (Spilochalcis) sp3 (femorata group; A. BOLD:ADE9827; B. BOLD:ADE9681); C \& D. Co. (Conura) sp6 (maculata group; C. BOLD:ADI0818; D. BOLD:ADE9680); E \& F. Co. (Sp.) sp18 (discolor group; E. BOLD:ADE9682; F. BOLD:ADE9822).

Some species had specimens recovered in different BINs, but were morphologically indistinct at first analysis, such as Co. sp3, Co. sp6, Co. sp9, Co. sp12 and Co. sp18 (Fig. 3). Further morphological analyses did reveal differences within Co. sp9 and Co. sp12, but no significant differences were found amongst Co. sp3, Co. sp6 and Co. sp18. Co. sp9 and Co. sp12 were then divided into Co. sp9 and Co. sp89 and Co. sp12 and Co. sp90, respectively. Co. sp9 and Co. sp89 differ in number and size of metafemur teeth, the first one with 13 and the second with 18 smaller, very close together and the gaster of $C$. sp9 is higher than in C. sp89. Conura sp12 and C. sp90 were more similar and harder to split, the only clear difference between these species was the lower face strigate in $C$. sp12 
and shallow fovea in $C$. sp90. This suggests that the DNA barcode may be a tool to refine taxonomy and even for detecting potential cryptic species of Chalcididae. The other three species remained morphologically indistinct, in spite of their different BINs. Perhaps further studies, including aspects of internal morphology, genitalia, biology and additional developmental data could help elucidating those cases.

Figure 4 shows a neighbour-joining distance tree (Fig. 4) with sequences longer than 200 bp and the subgenera of Conura highlighted with different colours. All subgenera, species complexes and several species groups were recovered as distinct groups. However, these results may suggest that the relationships within Conura do not fully correspond to the hypotheses previously proposed by Delvare 1992. Some morphology-based phylogenetic studies, developed at LaBI-UFES, also refute Delvare 1992. However, it remains to be demonstrated to what extent the fast-evolving $\mathrm{COI}$ gene is suitable for assessing higher level phylogenetic relationships within Chalcididae.

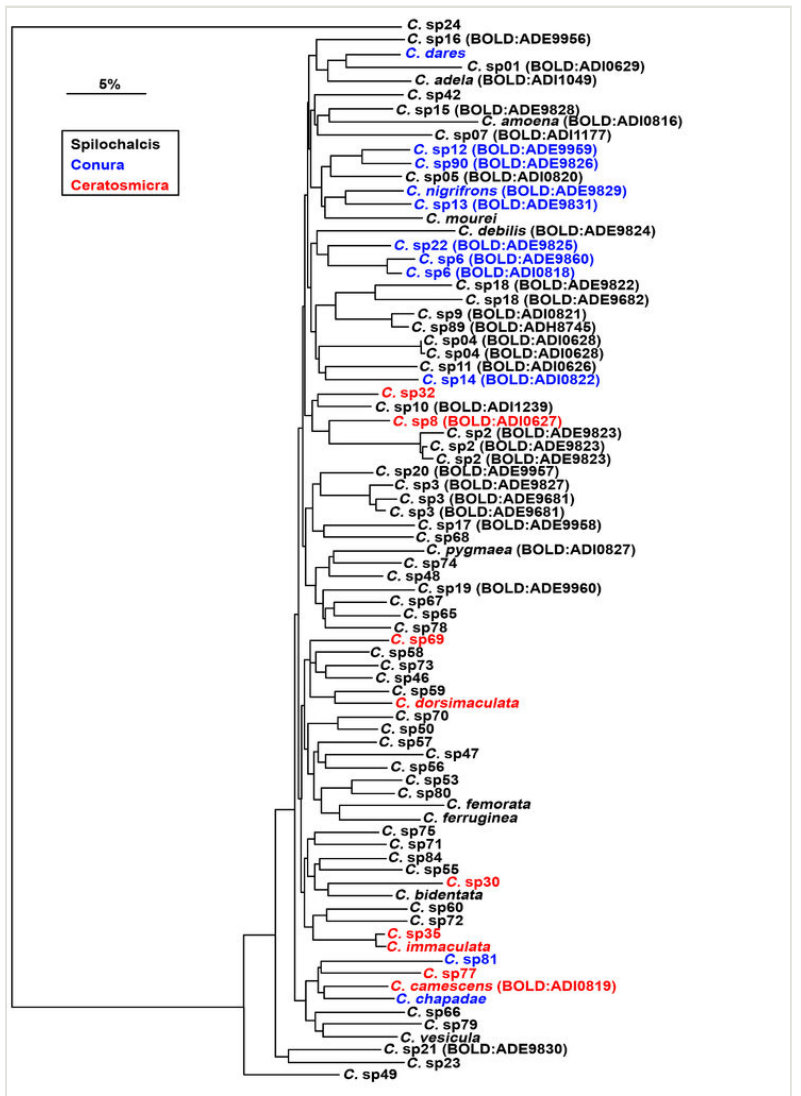

Figure 4. doi

A neighbour-joining distance tree of the Conura specimens with sequences longer than 200 bp. The colours represent subgenera. Available BINs are presented between brackets before the OTUs. 


\section{Melanosmicra Ashmead, 1904}

Melanosmicra is the only genus of the subfamily Chalcidinae found as new to Peru in this work. The number of specimens obtained with Malaise trap sampling (64 specimens or $15 \%$ of the total) was expressive (Table 4). Those specimens belong to 11 species, with three new species (Fig. 5). From this total, 21 specimens, representing all species found, were submitted to the molecular pipeline, producing 15 sequences longer than 100 $\mathrm{bp}$, which corresponded to four different BINs. The molecular findings were congruent with the morphological results. The numbers here reported put Peru as the second country in terms of number of species of Melanosmicra, with eight described species and three species new species, after Brazil, with 13 species recorded (Navarro-Tavares and Tavares 2008).

Table 4.

Melanosmicra specimens, BOLD data and corresponding BINs of the specimens submitted to the molecular pipeline.

\begin{tabular}{|l|l|l|l|}
\hline scientificName & Specimens & BIN & materialSampleID \\
\hline M. areta & 5 & ADF1337 & BC-ZSM-HYM-27511-B02; BC-ZSM-HYM-27511-B09 \\
\hline M. carenata & 2 & N/A & BC-ZSM-HYM-27511-B04; BC-ZSM-HYM-27511-B10 \\
\hline M. flavicollis & 1 & ADE9942 & BC-ZSM-HYM-27511-B11 \\
\hline M. gracilis & 6 & N/A & BC-ZSM-HYM-30556-B12; BC-ZSM-HYM-30556-C01 \\
\hline M. immaculata & 2 & ADF0815 & BC-ZSM-HYM-27511-B06; BC-ZSM-HYM-27511-C01; BC-ZSM- \\
HYM-27511-B01
\end{tabular}

\section{Brachymeria Westwood, 1829}

Brachymeria was represented by 67 specimens belonging to 14 species, 11 new species and three described (Table 5). Only the singleton $B$. sp 1 was collected in the fogging samples; the remainder were captured by Malaise trap. Brachymeria mnestor and $B$. pandora are frequently collected in the Neotropics. B. caudigera is very uncommon and it had been recorded so far only from the type locality (Jataí, State of Goiás, Brazil) (Fig. 6). 
Only four Brachymeria species had been previously recorded from Peru (Table 1). Therefore, with the records in this study, this number is increased to 18.

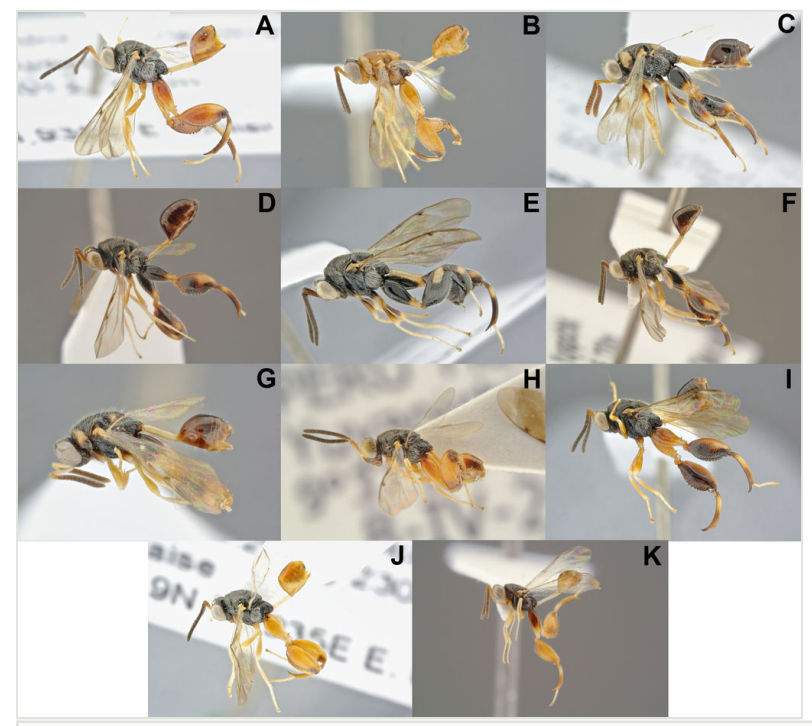

Figure 5. doi

Diversity of Melanosmicra. A. M. areta, female (BOLD:ADF1337); B. M. carenata, female; C. M. flavicollis, female (BOLD:ADE9942); D. M. gracilis, male; E. M. immaculata, male (BOLD:ADF0815); F. M. nigra, male; G. M. rugosa, female; H. M. tricolor, male; I. M. sp1, female (BOLD:ADF1769); J. M. sp2, female; K. M. sp3, male.

Table 5.

Brachymeria specimens, corresponding BINs and BOLD data of the specimens submitted to the molecular pipeline. An asterisk $\left({ }^{*}\right)$ indicates species collected using the fogging method.

\begin{tabular}{|l|l|l|l|}
\hline scientificName & Specimens & BIN & materialSampleID \\
\hline B. caudigera & 1 & N/A & BC-ZSM-HYM-25927-F10 \\
\hline B. mnestor & 13 & N/A & $\begin{array}{l}\text { BC-ZSM-HYM-25927-G06; BC-ZSM-HYM-25927-G10; BC-ZSM- } \\
\text { HYM-27511-F07 }\end{array}$ \\
\hline B. pandora & 37 & N/A & BC-ZSM-HYM-25927-G03; BC-ZSM-HYM-25927-F09 \\
\hline B. sp1 & $1^{*}$ & N/A & N/A \\
\hline B. sp2 & 1 & N/A & BC-ZSM-HYM-27511-F09 \\
\hline B. sp3 & 2 & ADI0920 & BC-ZSM-HYM-25927-G11; BC-ZSM-HYM-25927-G05 \\
\hline B. sp4 & 1 & ADH9819 & BC-ZSM-HYM-25927-G12 \\
\hline B. sp5 & 2 & ADF1042 & BC-ZSM-HYM-27511-F04 \\
\hline B. sp6 & 3 & N/A & BC-ZSM-HYM-25927-F07; BC-ZSM-HYM-25927-G04 \\
\hline
\end{tabular}




\begin{tabular}{|l|l|l|l|}
\hline scientificName & Specimens & BIN & materialSamplelD \\
\hline B. sp7 & 1 & N/A & BC-ZSM-HYM-25927-G11 \\
\hline B. sp8 & 3 & N/A & BC-ZSM-HYM-25927-G02; BC-ZSM-HYM-25927-G01 \\
\hline B. sp9 & 1 & N/A & BC-ZSM-HYM-25927-F06 \\
\hline B. sp10 & 1 & ADF1766 & BC-ZSM-HYM-27511-F05 \\
\hline B. sp11 & 1 & N/A & BC-ZSM-HYM-30556-H10 \\
\hline
\end{tabular}

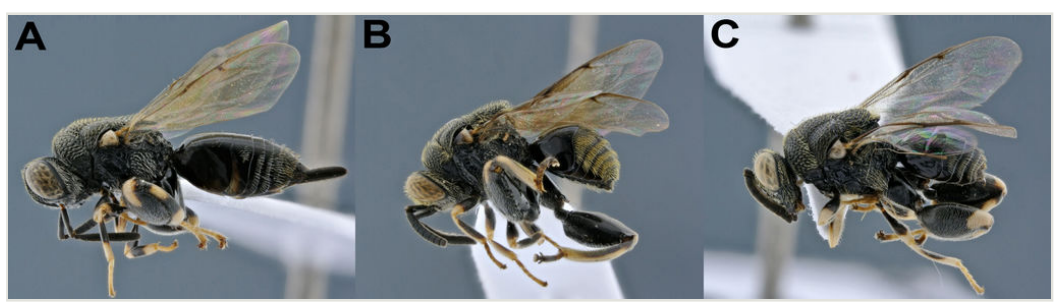

Figure 6. doi

Diversity of the Brachymeria described species: A. B. caudigera, female; B. B. mnestor, male; C. B. pandora, male.

Seventeen specimens, representing eleven species, were selected to be submitted to molecular pipeline and produced five sequences larger than $500 \mathrm{bp}$, resulting in four different BINs. Molecular and morphological results were congruent.

Brachymeria is distributed worldwide and includes about 307 described species (Noyes 2018). There are 46 described species in the Neotropical region (Noyes 2018) and at least 60 species awaiting description (unpublished data, MTT). Therefore, the Brachymeria fauna, sampled in Panguana, represents almost $10 \%$ of the Neotropical fauna.

\section{Ceyxia Girault, 1911}

Ceyxia was represented by five species and six specimens collected only with the Malaise trap (Table 6). Six species had previously been recorded for Peru: Ce. acutigaster, Ce. atuberculata, Ce. concitator, Ce. decreta, Ce. flaviscapus and Ce. villosa (Andrade and Tavares 2009). We recovered Ce. acutigaster and Ce. villosa and another three species: Ce. amazonica, Ce. bellissima and Ce. sp1. Thus, eight species are now known for the genus in Peru. Specimens of $\mathrm{Ce}$. acutigaster, Ce. amazonica and Ce. bellissima were submitted to the molecular pipeline, but no sequences were obtained (Fig. 7).

Table 6.

Ceyxia specimens and BOLD data of the specimens submitted to the molecular pipeline.

\begin{tabular}{|l|l|l|l|}
\hline scientificName & Specimens & BIN & materialSamplelD \\
\hline Ce. acutigaster & 1 & N/A & BC-ZSM-HYM-27511-F06 \\
\hline
\end{tabular}




\begin{tabular}{|l|l|l|l|}
\hline scientificName & Specimens & BIN & materialSampleID \\
\hline Ce. amazonica & 1 & N/A & BC-ZSM-HYM-27511-A12 \\
\hline Ce. bellissima & 1 & N/A & BC-ZSM-HYM-27511-F10 \\
\hline Ce. villosa & 1 & N/A & N/A \\
\hline Ce. $\mathrm{sp} 1$ & 1 & N/A & N/A \\
\hline
\end{tabular}

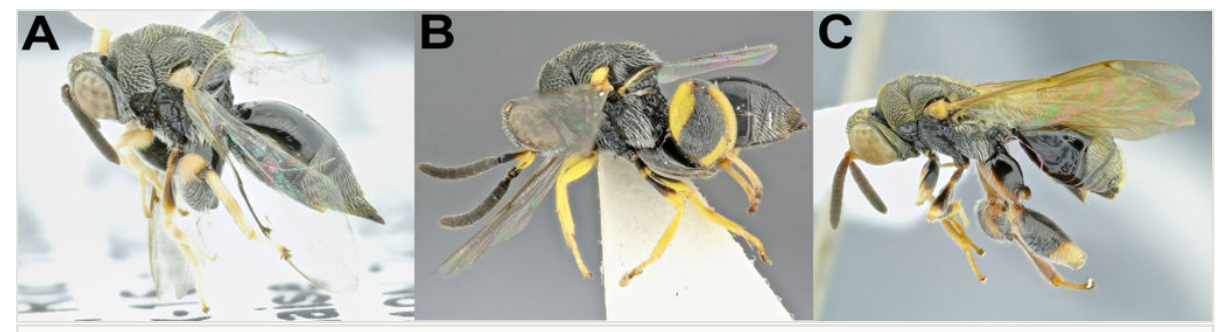

Figure 7. doi

Diversity of the Ceyxia described species, females: A. Ce. acutigaster; B. Ce. amazonica; C. Ce. bellissima.

\section{Stypiura Kirby, 1883}

A total of 18 specimens of Stypiura were obtained, representing 12 species (Table 7). According to Bouček (1992), amongst the genus of Phasgonophorini, only Stypiura had already been recorded from Peru. However, Bouček did not provide information on which species were found in Peru. In the present work, almost all recorded species are new. The only species found that had been previously described was $S$. batesii, which, so far, had not been recorded from Peru. Thus, 12 species are hereby recorded for that country: $S$. batesii, S. sp1, S. sp2, S. sp3, S. sp4, S. sp5, S. sp6, S. sp7, S. sp8, S. sp9, S. sp10 and S. sp11 (Fig. 8).

Table 7.

Stypiura specimens, coprresponding BINs and BOLD data of the specimens submitted to the molecular pipeline.

\begin{tabular}{|l|l|l|l|}
\hline scientificName & Specimens & BIN & materialSampleID \\
\hline S. batesii & 1 & N/A & BC-ZSM-HYM-27511-F03 \\
\hline S. sp1 & 1 & N/A & BC-ZSM-HYM-25927-E11 \\
\hline S. sp2 & 1 & N/A & BC-ZSM-HYM-27511-E07 \\
\hline S. sp3 & 1 & N/A & BC-ZSM-HYM-27511-E10 \\
\hline S. sp4 & 1 & N/A & BC-ZSM-HYM-27511-E08 \\
\hline S. sp5 & 1 & N/A & BC-ZSM-HYM-27511-E09 \\
\hline
\end{tabular}




\begin{tabular}{|l|l|l|l|}
\hline scientificName & Specimens & BIN & materialSampleID \\
\hline S. sp6 & 1 & BOLD:ADF0812 & $\begin{array}{l}\text { BC-ZSM-HYM-27511-F02; BC-ZSM-HYM-25927-E12; BC-ZSM- } \\
\text { HYM-30556-C11 }\end{array}$ \\
\hline S. sp7 & 1 & N/A & BC-ZSM-HYM-27511-E12 \\
\hline S. sp8 & 1 & N/A & $\begin{array}{l}\text { BC-ZSM-HYM-27511-F01; BC-ZSM-HYM-25927-F02; BC-ZSM- } \\
\text { HYM-25927-F03; BC-ZSM-HYM-30556-C12 }\end{array}$ \\
\hline S. sp9 & 1 & N/A & BC-ZSM-HYM-27511-E11 \\
\hline S. sp10 & 1 & N/A & N/A \\
\hline S. sp11 & 3 & N/A & BC-ZSM-HYM-25927-F01; BC-ZSM-HYM-30556-C10 \\
\hline
\end{tabular}

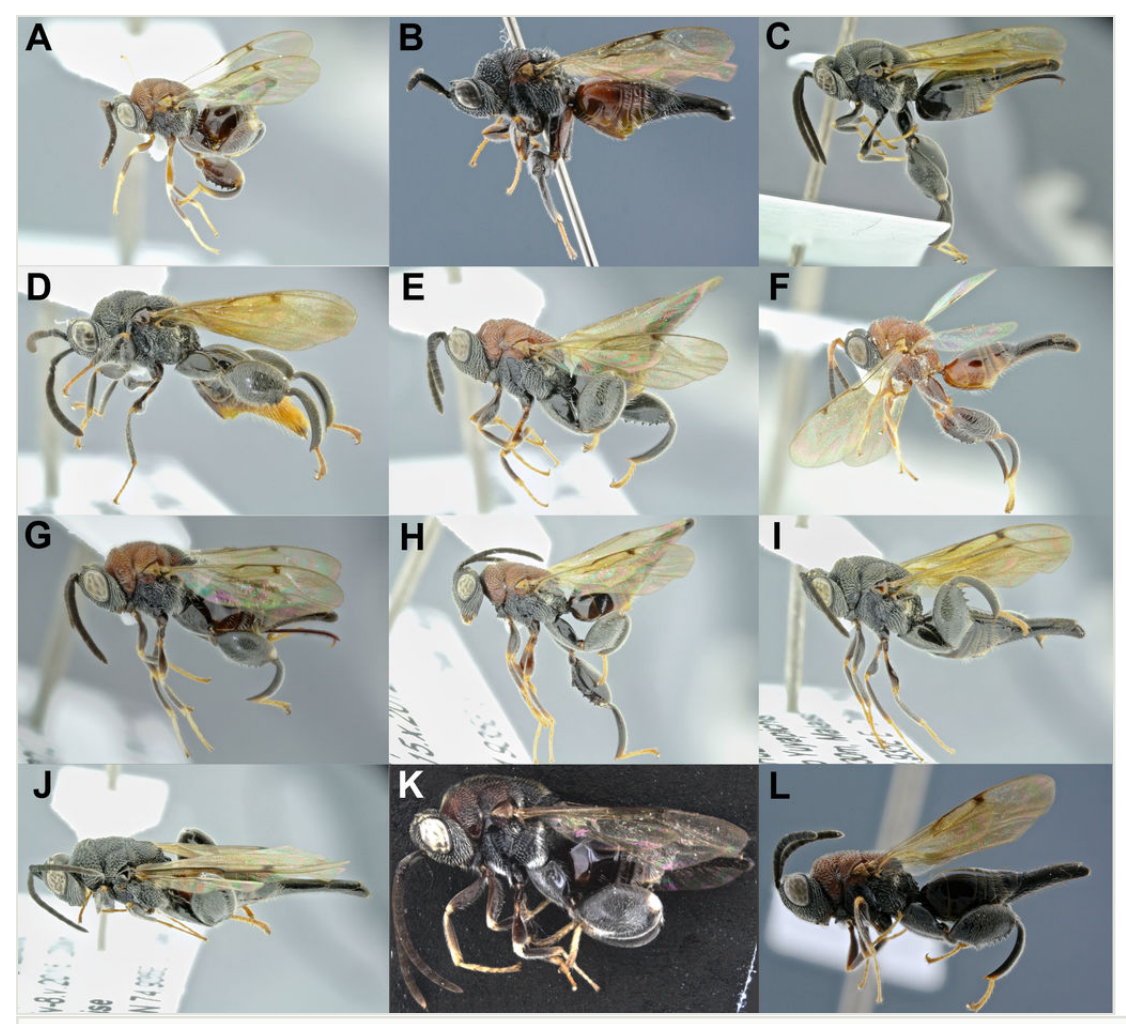

Figure 8. doi

Diversity of Stypiura, females. A. S. batesii; B. S. sp1; C. S. sp2; D. S. sp3; E. S. sp4; F. S. sp5; G. S. sp6 (BOLD:ADF0812); H. S. sp7; I. S. sp8; J. S. sp9; K. S. sp10 ; L. S. sp11.

The most abundant species were $S$. sp6 and $S$. sp8, with 3 and 4 specimens, respectively. The species S. batesii, S. sp2, S. sp6 and S. sp7 were chosen for the molecular pipeline. Despite the cluster being congruent with the morphological delimitation of the species, the only species that received a BIN was S. sp6. 


\section{Dirhinus Dalman, 1818}

The current classification of Dirhinus presents three subgenera: Dirhinus, Hontalia and Pareniaca, all found in the New World; the second is exclusively neotropical. Although it is quite diverse for the New World (16 species), the only species known from Peru is $D$. (Dirhinus) giffardii, which was not found in the studied samples. After morphological analysis of the 28 specimens obtained, we were able to recognise nine species (Fig. 9). Eight species belong to subgenus Pareniaca (six new to science) and one species belongs to the subgenus Hontalia. We submitted 18 specimens, representing all species found of Dirhinus, to the molecular pipeline. Ten produced sequences longer than $100 \mathrm{bp}$ and were included in a cluster analysis (Table 8). The clusters presented an average of $6.05 \%$ of nearest neighbour distance (NN), with the clustering fully corresponding with the results of the morphological analysis. Seven BINs were associated with the successfully sequenced samples, all new to BOLD (Table 8). These seven new species correspond to an increase of $43.7 \%$ in the Dirhinus diversity known from the New World.

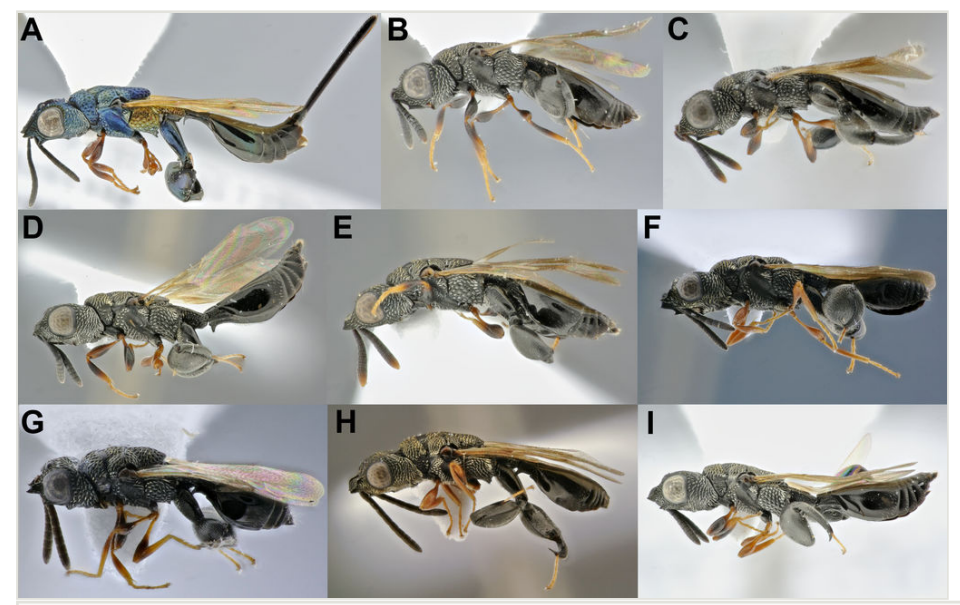

Figure 9. doi

Diversity of Dirhinus, females, except $\mathrm{F}$ and $\mathrm{H}$, males. A. D. cameroni; B. D. buscki (BOLD: ADF1234); C. D. kirbyi; $\quad$ D. D. sp1; $\quad$ E. D. sp2; $\quad$ F. D. sp3; G. D. sp4; H.D. sp5; I. D. sp6 (BOLD: ADE9797)

Table 8.

Dirhinus specimens, corresponding BINs and BOLD data of the specimens submitted to the molecular pipeline.

\begin{tabular}{|l|l|l|l|}
\hline scientificName & Specimens & BIN & materialSampleID \\
\hline D. buscki & 4 & ADF1234 & $\begin{array}{l}\text { BC-ZSM-HYM-27511-C03; BC-ZSM-HYM-27511-C09; BC-ZSM- } \\
\text { HYM-27511-C11 }\end{array}$ \\
\hline D. cameroni & 8 & N/A & $\begin{array}{l}\text { BC-ZSM-HYM-25927-E02; BC-ZSM-HYM-27511-C02; BC-ZSM- } \\
\text { HYM-30556-B01 }\end{array}$ \\
\hline
\end{tabular}




\begin{tabular}{|l|l|l|l|}
\hline scientificName & Specimens & BIN & materialSampleID \\
\hline D. kirbyi & 4 & ADE9658 & $\begin{array}{l}\text { BC-ZSM-HYM-27511-C04; BC-ZSM-HYM-27511-C08; BC-ZSM- } \\
\text { HYM-27511-C10 }\end{array}$ \\
\hline D. sp1 & 4 & N/A & $\begin{array}{l}\text { BC-ZSM-HYM-25927-E03; BC-ZSM-HYM-27511-C07; BC-ZSM- } \\
\text { HYM-30556-B02 }\end{array}$ \\
\hline D. sp2 & 1 & N/A & BC-ZSM-HYM-25927-E05 \\
\hline D. sp3 & 1 & N/A & BC-ZSM-HYM-25927-E04 \\
\hline D. sp4 & 4 & N/A & BC-ZSM-HYM-30556-B03 \\
\hline D. sp5 & 1 & N/A & BC-ZSM-HYM-27511-C05 \\
\hline D. sp6 & 1 & ADE9797 & BC-ZSM-HYM-27511-C06 \\
\hline
\end{tabular}

\section{Notaspidium Dalla Torre, 1897}

Notaspidium is the only genus of Haltichellinae previously recorded from Peru. It was known from two species ( $N$. apantelis and $N$. giganteum). Only $N$. apantelis was obtained in the present study. Notaspidium was the dominant genus in the fogging samples, with 11 specimens and 8 species (Table 9). A total of 37 specimens and 12 species were found in the morphological analysis (Fig. 10), four of them new species. Eighteen specimens and 12 species were selected to the molecular pipeline. Eleven specimens produced sequences longer than $100 \mathrm{bp}$ and were included in a cluster analysis (Table 2). The clusters presented an average of $11.26 \%$ nearest neighbour (NN) distance, fully matching the results of the morphological analysis. The four BINs found are new to BOLD (Table 9).

Table 9.

Notaspidium specimens, corresponding BINs and BOLD data of the specimens submitted to the molecular pipeline. Brackets indicate the number of specimens collected using the fogging method.

\begin{tabular}{|c|c|c|c|}
\hline scientificName & Specimens & BIN & materialSampleID \\
\hline N. acutum & 5 & ADF1074 & BC-ZSM-HYM-27511-D10 \\
\hline N. apantelis & $1(1)$ & $\mathrm{N} / \mathrm{A}$ & BC-ZSM-HYM-30556-A07 \\
\hline N. boharti & $2(1)$ & ADF1075 & $\begin{array}{l}\text { BC-ZSM-HYM-30556-A11 } \\
\text { BC-ZSM-HYM-27511-D11 }\end{array}$ \\
\hline N. braziliensis & $2(2)$ & $\mathrm{N} / \mathrm{A}$ & BC-ZSM-HYM-30556-A08 \\
\hline N. burdicki & $13(2)$ & $\mathrm{N} / \mathrm{A}$ & $\begin{array}{l}\text { BC-ZSM-HYM-27511-E01 } \\
\text { BC-ZSM-HYM-27511-D12 } \\
\text { BC-ZSM-HYM-25927-E07 } \\
\text { BC-ZSM-HYM-25927-E06 }\end{array}$ \\
\hline N. minutum & $3(1)$ & ADE9953 & BC-ZSM-HYM-27511-E03 \\
\hline
\end{tabular}




\begin{tabular}{|l|l|l|l|}
\hline scientificName & Specimens & BIN & materialSamplelD \\
\hline N. truncatum & 2 & N/A & $\begin{array}{l}\text { BC-ZSM-HYM-25927-E08 } \\
\text { BC-ZSM-HYM-27511-E04 }\end{array}$ \\
\hline N. villegasi & 1 & N/A & BC-ZSM-HYM-27511-E05 \\
\hline N. sp1 & $3(2)$ & N/A & BC-ZSM-HYM-30556-A09 \\
\hline N. sp2 & 2 & ADJ2148 & BC-ZSM-HYM-27511-E06 \\
\hline N. sp3 & $1(1)$ & NC-ZSM-HYM-25927-E09 \\
\hline N. sp4 & $2(1)$ & N/A & BC-ZSM-HYM-30556-A12 \\
\hline
\end{tabular}

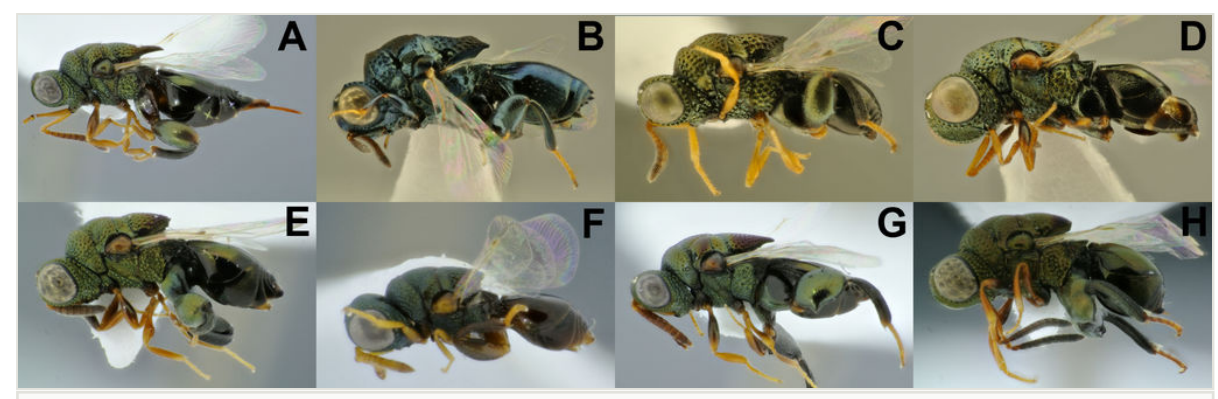

Figure 10. doi

Diversity of the Notaspidium described species: A. N. acutum, female; B. N. apantelis, female; C. N. boharti, male; D. N. braziliensis; E. N. burdicki; F. N. minutum; G. N. truncatum; H. N. villegsasi

\section{Aspirrhina Kirby, 1883}

This is the first time that the genus Aspirrhina has been recorded from Peru. Three species (A. remotor, A. bifurca and A. dubitator) were identified, based on 12 specimens (Fig. 11). Aspirrhina bifurca was the most common species, with 8 specimens. Eight specimens representing all putative taxa found were submitted to the molecular pipeline, but no sequences were obtained.
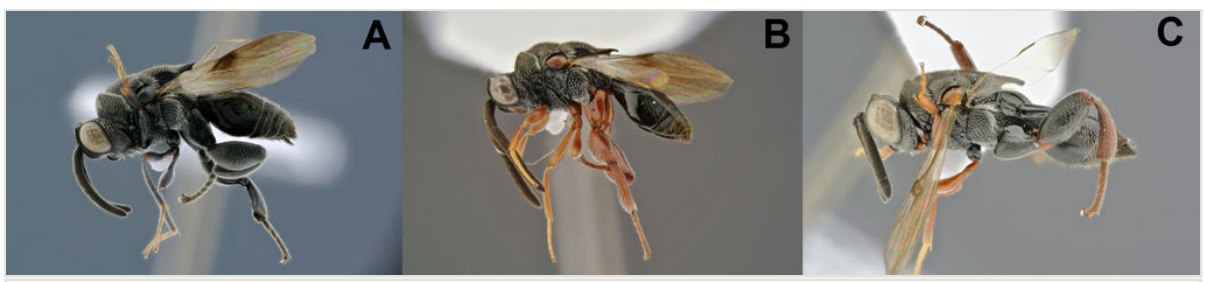

Figure 11. doi

Diversity of Aspirrhina. A. A. remotor, male; B. A. bifurca, male; C. A. dubitator, female. 


\section{Haltichella Spinola, 1811}

Although it is one of the most common genera obtained in neotropical Malaise trap samples, along with Conura and Brachymeria, this is the first time Haltichella has been recorded from Peru. Twenty-seven specimens were collected corresponding to four new species (Fig. 12). One species (Ha. sp3) was represented by 24 specimens and all others were singletons. Fourteen specimens were submitted to the molecular pipeline (Table 10). Only two specimens (representing $\mathrm{Ha}$. sp3 and $\mathrm{Ha}$. sp4) had the COI-5P fragment successfully amplified, but the sequences generated did not meet the criteria for BIN assignment. These specimens formed two separate clusters, which were congruent with the morphological analysis.

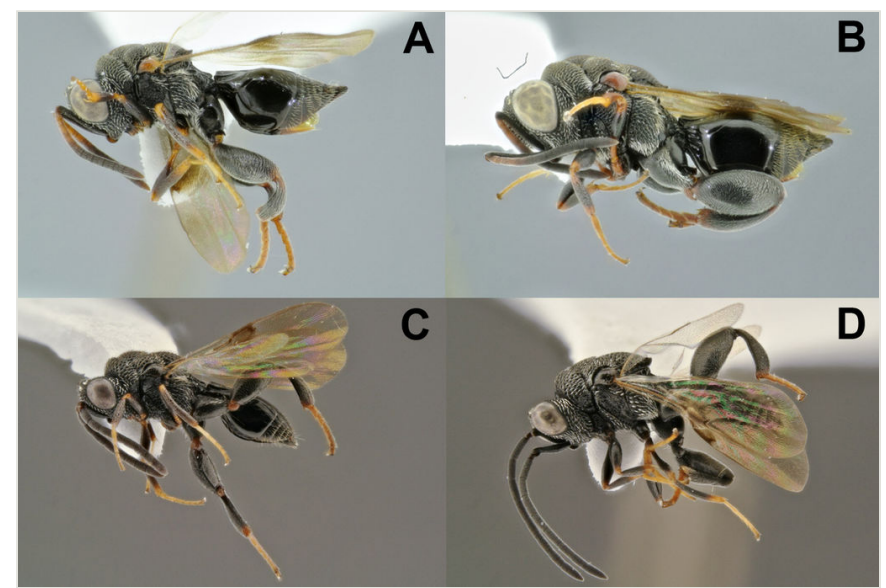

Figure 12. doi

Diversity of Haltichella. A. Ha. sp1, female; B. Ha. sp2, female; C. Ha. sp3, male; D. Ha. sp4, male.

Table 10.

Haltichella specimens, corresponding BINs and BOLD data of the specimens submitted to the molecular pipeline.

\begin{tabular}{|c|c|c|c|}
\hline scientificName & Specimens & BIN & materialSampleID \\
\hline H. sp1 & 1 & $\begin{array}{l}\mathrm{N} / \\
\mathrm{A}\end{array}$ & BC-ZSM-HYM-27511-D02 \\
\hline H. sp2 & 24 & N/A & $\begin{array}{l}\text { BC-ZSM-HYM-27511-D09; BC-ZSM-HYM-27511-D08; BC-ZSM-HYM-27511-D07; } \\
\text { BC-ZSM-HYM-27511-D06; BC-ZSM-HYM-27511-D03; BC-ZSM-HYM-27511-D04; } \\
\text { BC-ZSM-HYM-27511-D05; BC-ZSM-HYM-25927-D08; BC-ZSM-HYM-25527-D09; } \\
\text { BC-ZSM-HYM-25927-D10; BC-ZSM-HYM-30556-A01 }\end{array}$ \\
\hline H. sp3 & 1 & N/A & BC-ZSM-HYM-30556-A02 \\
\hline H. sp4 & 1 & $\mathrm{~N} / \mathrm{A}$ & BC-ZSM-HYM-30556-A03 \\
\hline
\end{tabular}




\section{Hockeria Walker, 1834}

Hockeria is here recorded for the first time from Peru and was represented by two specimens of the same species (Fig. 13). Those two males do not seem to correspond to any known neotropical species. Both specimens were submitted to the molecular pipeline, but no sequences were obtained.

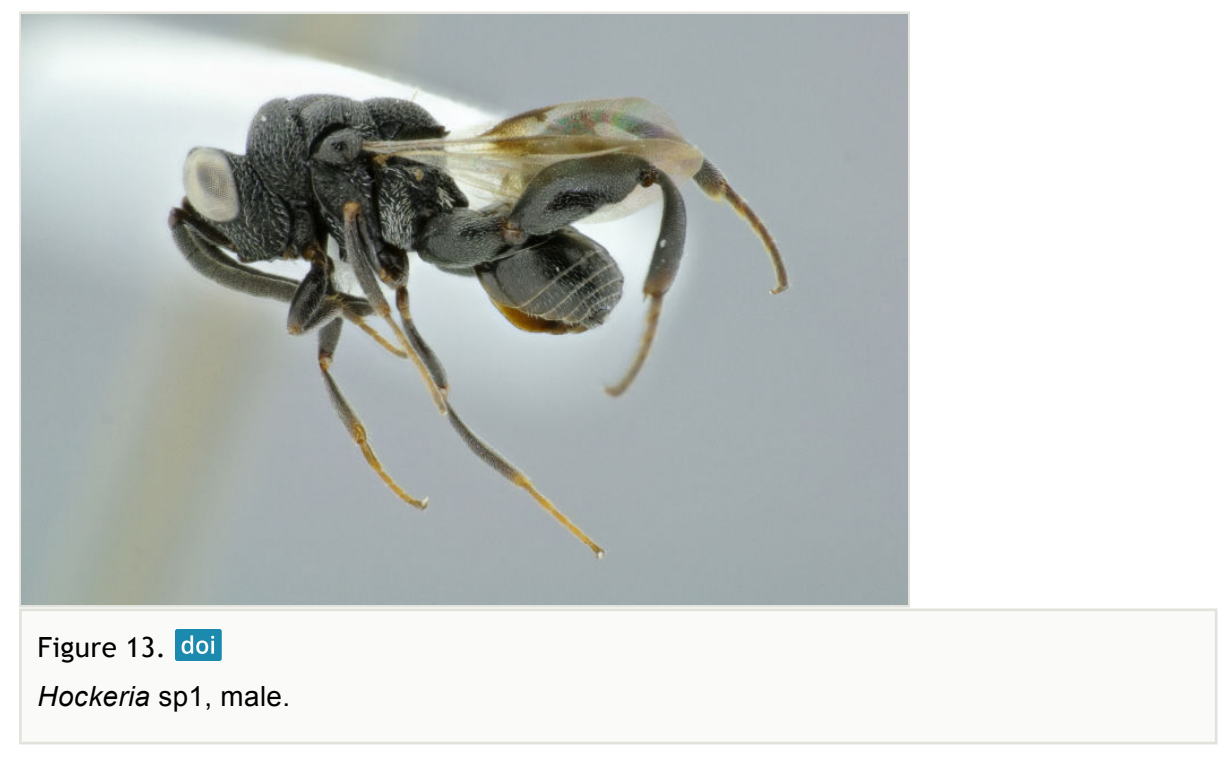

\section{Discussion}

This paper presents the largest inventory ever made for the Peruvian Amazon fauna of Chalcididae and it provides new data which may drive future studies.

The abundance and diversity of species of Chalcididae in Panguana present similarities and divergences when compared with long term Malaise samplings from other Amazonian regions (housed at traditional Amazonian Institution in Brazil; unpublished data, MTT). Conura is usually the richest and more abundant genus in these samples, followed by Brachymeria, which is the case of the present samples. However, in the Panguana samples, Melanosmicra, Notaspidium and Stypiura were also diverse in species, similar to Brachymeria. Melanosmicra and Stypiura are usually captured in Malaise samples from the Amazonian forest, but they are not as diverse as in the Panguana samples. In forested areas, Notaspidium is not common in Malaise samples collected at soil level, although they are frequent in canopy samples obtained with interception traps (Malaise and window traps) and fogging. In Panguana, Notaspidium was well represented in the canopy samples, as well as at soil level. Haltichella tends to be frequent and abundant in samples of open vegetation (such as savannah and partially deforested areas), but not in forested areas. In Panguana, the genus was unexpectedly abundant, besides the relative high richness. All the above aspects indicate that the 
Chalcididae fauna of Panguana is peculiar and efforts should be made to better understand its fauna.

There are 386 Hymenoptera records publicly available on BOLD for Peru but none for Chalcididae. The 207 records presented here are the first records for Peruvian Chalcididae in the BOLD database. A total of 60 sequences were associated with a BIN, corresponding to 50 unique BINs, meaning that 81 specimens with sequences did not receive a BIN. This large number of specimens with sequences without a BIN was due to short sequences (around $401 \mathrm{bp}$ ). Given that most of those species are new to BOLD (and to science), in this case, the sequences were required to have a minimum of $500 \mathrm{bp}$ to generate a new $\mathrm{BIN}$ in the system.

The sorted species, based on morphological data (morphospecies), were very consistent with those based on molecular data (DNA barcodes). While morphology indicated a total of 118 species, the barcodes indicated 121 species (sequences longer than $100 \mathrm{bp}$ ), which suggested three couples of cryptic species. This fact indicates that very few cryptic species are present in the sample and that both methods seem to be appropriate to study the diversity of Chalcididae fauna.

The 10 genera and 183 species obtained from the estimated 500 trap-days sampling effort demonstrate the high richness present in Panguana. Besides these species, one genus and twelve described species have been recorded to Peruvian Amazon (Table 1). Therefore, at least 11 genera and 195 species are present in Peruvian Amazon. This species richness is higher than the 149 species (described and undescribed) known from the State of Espírito Santo, Brazil (Tavares and de Araujo 2007), which is located in the Atlantic Forest, another biodiversity hotspot.

So far, only 7 genera and 11 described species had been recorded from the Peruvian Amazon. The present study adds 4 genera and 39 species, totalling 11 genera and 50 described species. Country-wise, the fauna of described species of Peruvian Chalcididae is now 13 genera and 76 described species, which is still less than 96 species reported from Colombia by Arias and Delvare (2003).

From the total of 183 species found here, 113 are potentially new species. Most of those species were represented by singletons, giving a clear picture of the potential diversity of the Peruvian Amazon and highlighting the importance of initiatives like the Panguana reserve.

We also draw attention to the importance of international collaboration initiatives, like the partnership established between ZSM and UFES, supporting the high-speed exchange of expertise and infrastructure, culminating in fast and high-quality results in taxonomy.

\section{Acknowledgements}

We would like to thank Juliane and Erich Diller for their support and for allowing us to study specimens of Chalcididae collected at the Panguana station in Peru. We are grateful for 
permits by MINAET for research at the Panguana reserve, conducted under the "Agreement of Scientific Cooperation between The Natural History Museum of the National University of San Marcos, Lima, Peru, and the Bavarian Natural History Collections, Munich, Germany" and with kind permission of the Servicio Nacional Forestal y de Fauna Silvestre SERFOR (Ministry of Agriculture), Lima, Peru (collecting permits (2003-2017): No. 0/6-2003-INRENA-IFFS-DCB to No. 007-2014-SERFOR-DGGSPFFS (permit for 5 years) + No. 0406-2017-SERFOR-DGGSPFFS, export permits (2003-2017): No. 0002376AG-INRENA to No. 003236-SERFOR). We would like to thank Sajad Noori and Ana Maria Bastidas Urrutia for their support with the specimens imaging. The sequence analyses for this study were supported, in part, by Genome Canada through the Ontario Genomics Institute, while informatics support was provided through a grant from the Ontario Ministry of Research and Innovation. The authors thank Fundação de Amparo à Pesquisa e Inovação do Espírito Santo (FAPES, Proc \# 67658830/2014), Instituto Nacional de Ciência e Tecnologia dos Hymenoptera Parasitoides (CNPq, proc. \#465562/2014-0; FAPESP \#2014/50940-2) and Coordenação de Aperfeiçoamento de Pessoal de Nível Superior Brasil (CAPES, Finance Code 001), for the financial support. PMS thanks CAPES for the doctoral research grant (proc. \#88887.352332/2019-00). TRAB thanks CAPES for the doctoral research grant (proc. \#8887.137875/2017-00). JMS-F thanks FAPES for the doctoral research grant (proc. \#69918333/2015-00). MEVS thanks CAPES for the doctoral research grant (proc. \#1504421/2015 and 1702180/2015). We also would like to thank Ana Dal Molin (Laboratório de Escrita Científica) for proofreading and reviewing an early version of the manuscript, the journal editor and the peer reviewers.

\section{References}

- $\quad$ Andrade TVd, Tavares MT (2009) Revisão de Ceyxia Girault, stat. rev. (Hymenoptera, Chalcididae, Brachymeriini). Revista Brasileira de Entomologia 53 (4): 511-548. https:// doi.org/10.1590/s0085-56262009000400004

- Arias D, Delvare G (2003) Lista de los géneros y espécies de la família Chalcididae (Hymenoptera: Chalcidoidea) de la región Neotropical. Biota Colombiana 4 (2): 123-145. https://doi.org/10.21068/bc.v4i2.129

- $\quad$ Asner G, Martin R, Tupayachi R, Llactayo W (2017) Conservation assessment of the Peruvian Andes and Amazon based on mapped forest functional diversity. Biological Conservation 210: 80-88. https://doi.org/10.1016/j.biocon.2017.04.008

- $\quad$ Bouček Z (1992) The New World genera of Chalcididae . In: Delvare G, Bouček Z (Eds) On the New World Chalcididae (Hymenoptera). 1. Gainesville, 466 pp. [ISBN 1-56665-053-4].

- $\quad$ Chavez A (2013) Landscape dynamics of Amazonian deforestation between 1986 and 2007 in southeastern Peru: policy drivers and road implications. Journal of Land Use Science 9 (4): 414-437. https://doi.org/10.1080/1747423x.2013.807310

- Delvare G (1992) A reclassification of the Chalcidini with a checklist of the New World species. In: Gerard Delvare, Zdenek Bouček On the New World Chalcididae (Hymenoptera). 1, 1. Associated Publishers, Gainesville, Florida, 466 pp. [ISBN 1-56665-053-4]. 
- Delvare G (1997) Description de Conura initia n.sp. d'Amérique tropicale (Hymenoptera, Chalcididae). Bulletin de la Société Entomologique de France 101 (5): 500-50.

- Delvare G (2006) Chalcididae. In: Hanson PE, Gauld ID (Eds) Hymenoptera de La Región Neotropical. 1. The American Entomological Institute, Gainesville, 994 pp. [ISBN 1-887988-21-1].

- Delvare G, Huchet J (2017) Brachymeria mochica, a new Neotropical species of Chalcididae (Hymenoptera: Chalcidoidea) discovered on the archaeological site of Huacas de Moche, Peru with a review of related species. Zootaxa 4290 (1): 43. https:// doi.org/10.11646/zootaxa.4290.1.2

- Delvare G, Hannes Baur GH, Chadee D, Martinez R, Knutie S (2017) Description of Brachymeria philornisae sp. n. (Hymenoptera: Chalcididae), a parasitoid of the bird parasite Philornis trinitensis (Diptera: Muscidae) in Tobago, with a review of the sibling species. Zootaxa 4242 (1). https://doi.org/10.11646/zootaxa.4242.1.2

- De Santis L (1979) Catálogo de los himénopteros calcidoideos de América al sur de los Estados Unidos. Publicación Especial Comisión de Investigaciones Cientificas Provincia de Buenos Aires, 302 pp.

- deWaard J, Ivanova N, Hajibabaei M, Hebert PN (2008) Assembling DNA barcodes. Methods in Molecular Biology. https://doi.org/10.1007/978-1-59745-548-0_15

- Erwin T, Hillis D, Fonseca G, Kelly K, Mittermeier R, Prance G, Phelan R, Soberon J, Warshall P (2007) ALL species foundation. https://web.archive.org/web/20070202032924/ http://www.all-species.org/call.html. Accessed on: 2019-9-07.

- $\quad$ Floren A (2010) Sampling arthropods from the canopy by insecticidal knockdown. In: Eymann J, Degreef J, Häuser C, Monje JC, Samyn Y, Vandenspiegel D (Eds) Manual on field recording techniques and protocols for all taxa biodiversity inventories. 1, 8. ABC Taxa, Brussels, 653 pp. URL: http://www.abctaxa.be/volumes/volume-8-manual-atbi/ chapter-8/Chapter 8.pdf/download/en/1/Chapter\%208.pdf?action=view

- Halstead JA (1991) New species of Notaspidium Dalla Torre from the Nearctic and Neotropical regions (Hymenoptera: Chalcididae). Pan-Pacific Entomologist 67 (4): 232-235.

- Hebert PDN, Cywinska A, Ball SL, deWaard JR (2003) Biological identifications through DNA barcodes. Proceedings of the Royal Society B: Biological Sciences 270 (1512): 313-321. https://doi.org/10.1098/rspb.2002.2218

- Ivanova N, deWaard J, Hebert PN (2006) An inexpensive, automation-friendly protocol for recovering high-quality DNA. Molecular Ecology Notes 6 (4): 998-1002. https:// doi.org/10.1111/j.1471-8286.2006.01428.x

- $\quad$ Kimura M (1980) A simple method for estimating evolutionary rates of base substitutions through comparative studies of nucleotide sequences. Journal of Molecular Evolution 16: 111-120. https://doi.org/10.1007/BF01731581

- $\quad$ Mittermeier RA, Robles G, Mittermeier CG (1999) Megadiversity: Earth's biologically wealthiest nations. Biodiversity a-z URL: https://www.environment.gov.za/sites/default/files/ docs/cancun Immc declaration1.pdf

- $\quad$ Myers N, Mittermeier R, Mittermeier C, da Fonseca GB, Kent J (2000) Biodiversity hotspots for conservation priorities. Nature 403 (6772): 853-858. https:// doi.org/10.1038/35002501

- $\quad$ Navarro-Tavares AB, Tavares MT (2008) Revisão das espécies de Melanosmicra Ashmead (Hymenoptera, Chalcididae). Revista Brasileira de Entomologia 52 (4): 550-574. https://doi.org/10.1590/s0085-56262008000400005 
- $\quad$ Noyes JS (2018) Universal Chalcidoidea Database. http://www.nhm.ac.uk/our-science/ data/chalcidoids/database/. Accessed on: 2018-11-05.

- Ratnasingham S, Hebert PN (2007) BARCODING: bold: The Barcode of Life Data System (http://www.barcodinglife.org). Molecular Ecology Notes 7 (3): 355-364. https:// doi.org/10.1111/j.1471-8286.2007.01678.x

- $\quad$ Ratnasingham S, Hebert PN (2013) A DNA-based registry for all animal species: The Barcode Index Number (BIN) System. PLOS One 8 (7): e66213. https://doi.org/10.1371/ journal.pone.0066213

- $\quad$ Tavares MT, de Araujo BC (2007) Espécies de Chalcididae (Hymenoptera, Insecta) do Estado do Espírito Santo, Brasil. Biota Neotropica 7 (2): 213-220. https://doi.org/10.1590/ s1676-06032007000200024

- $\quad$ Tavares MT, Aquino D (2014) Chalcididae. In: Roig-Juñent S, Claps L, Morrone J (Eds) Biodiversidad de artrópodos argentinos. 1, 4. Universidad Nacional de Tucumán. Facultad de Ciencias Naturales, San Miguel de Tucumán, 548 pp. [ISBN 978-950-554-905-4].

- $\quad$ Tavares MT, Villanueva-Bonilla GA, Sobczak JF (2019) Conura baturitei sp. nov. (Hymenoptera: Chalcididae): a hyperparasitoid of spiders through Zatypota riverai (Hymenoptera: Ichneumonidae). Plazi.org taxonomic treatments database https:// doi.org/10.15468/MTALOB

- $\quad$ Townes H (1972) A light-weight Malaise trap. Entomological News 83 (9): 239-247.

\section{Supplementary material}

\section{Suppl. material 1: Peruvian Chalcididae summary table doi}

Authors: Bruno Cancian et al.

Data type: Excel sheet

Download file $(24.74 \mathrm{~kb})$ 